\title{
CARLESON MEASURES FOR WEIGHTED HARDY-SOBOLEV SPACES
}

\author{
CARME CASCANTE AND JOAQUIN M. ORTEGA
}

\begin{abstract}
We obtain characterizations of positive Borel measures $\mu$ on $\mathbf{B}^{n}$ so that some weighted Hardy-Sobolev are imbedded in $L^{p}(d \mu)$, where $w$ is an $A_{p}$ weight in the unit sphere of $\mathbf{C}^{n}$.
\end{abstract}

\section{$\S 1$. Introduction}

The purpose of this paper is the study of the positive Borel measures $\mu$ on $\mathbf{S}^{n}$, the unit sphere in $\mathbf{C}^{n}$, for which the weighted Hardy-Sobolev space $H_{s}^{p}(w)$ is imbedded in $L^{p}(d \mu)$, that is, the Carleson measures for $H_{s}^{p}(w)$.

The weighted Hardy-Sobolev space $H_{s}^{p}(w), 0<s, p<+\infty$, consists of those functions $f$ holomorphic in $\mathbf{B}^{n}$ such that if $f(z)=\sum_{k} f_{k}(z)$ is its homogeneous polynomial expansion, and $(I+R)^{s} f(z)=\sum_{k}(1+k)^{s} f_{k}(z)$, we have that

$$
\|f\|_{H_{s}^{p}(w)}=\sup _{0<r<1}\left\|(I+R)^{s} f_{r}\right\|_{L^{p}(w)}<+\infty
$$

where $f_{r}(\zeta)=f(r \zeta)$.

We will consider weights $w$ in $A_{p}$ classes in $\mathbf{S}^{n}, 1<p<+\infty$, that is, weights in $\mathbf{S}^{n}$ satisfying that there exists $C>0$ such that for any nonisotropic ball $B \subset \mathbf{S}^{n}, B=B(\zeta, r)=\left\{\eta \in \mathbf{S}^{n} ;|1-\zeta \bar{\eta}|<r\right\}$,

$$
\left(\frac{1}{|B|} \int_{B} w d \sigma\right)\left(\frac{1}{|B|} \int_{B} w^{\frac{-1}{p-1}} d \sigma\right)^{p-1} \leq C,
$$

where $\sigma$ is the Lebesgue measure on $\mathbf{S}^{n}$ and $|B|$ the Lebesgue measure of $B$. We will use the notation $\zeta \bar{\eta}$ to indicate the complex inner product in $\mathbf{C}^{n}$ given by $\zeta \bar{\eta}=\sum_{i=1}^{n} \zeta_{i} \bar{\eta}_{i}$, if $\zeta=\left(\zeta_{1}, \ldots, \zeta_{n}\right), \eta=\left(\eta_{1}, \ldots, \eta_{n}\right)$.

Received January 12, 2005.

1991 Mathematics Subject Classification: 32A35, 46E35, 32A40.

Both authors partially supported by DGICYT Grant MTM2005-08984-C02-02, and DURSI Grant 2005SGR 00611. 
If $0<s<n$, any function $f$ in $H_{s}^{p}(w)$ can be expressed as

$$
f(z)=C_{s}(g)(z):=\int_{\mathbf{S}^{n}} \frac{g(\zeta)}{(1-z \bar{\zeta})^{n-s}} d \sigma(\zeta),
$$

where $d \sigma$ is the normalized Lebesgue measure on the unit sphere $\mathbf{S}^{n}$ and $g \in L^{p}(w)$, and consequently, $\mu$ is Carleson for $H_{s}^{p}(w)$ if there exists $C>0$ such that

$$
\left\|C_{s} f\right\|_{L^{p}(d \mu)} \leq C\|f\|_{L^{p}(w)} .
$$

We denote by $K_{s}$ the nonisotropic potential operator defined by

$$
K_{s}[f](z)=\int_{\mathbf{S}^{n}} \frac{f(\eta)}{|1-z \bar{\eta}|^{n-s}} d \sigma(\eta), \quad z \in \overline{\mathbf{B}}^{n} .
$$

The problem of characterizing the positive Borel measures $\mu$ on $\mathbf{B}^{n}$ for which there exists $C>0$ such that

$$
\left\|K_{s}[f]\right\|_{L^{p}(d \mu)} \leq C\|f\|_{L^{p}(d \sigma)},
$$

that is, the characterization of the Carleson measures for the space $K_{s}\left[L^{p}(d \sigma)\right]$ has been very well studied and there exist different characterizations (see for instance [Ma], [AdHe], [KeSa]).

The representation of the functions in $H_{s}^{p}$ in terms of the operator $C_{s}$ gives that in dimension 1 the Carleson measures for $K_{s}\left[L^{p}(d \sigma)\right]$ coincide with the Carleson measures for the Hardy-Sobolev space $H_{s}^{p}$ simply because the real part of $1 /(1-z \bar{\zeta})^{1-s}$ is equivalent to $1 /|1-z \bar{\zeta}|^{1-s}$. This representation also shows that in any dimension every Carleson measure for $K_{s}\left[L^{p}(d \sigma)\right]$ is also a Carleson measure for $H_{s}^{p}$. The coincidence fails to be true for $n>1$ in general, as it is shown in $[\mathrm{AhCo}]$ (see also [CaOr2]).

Of course, when $n-s p<0$, the space $H_{s}^{p}$ consists of continuous functions on $\overline{\mathbf{B}}^{n}$, and in particular, the Carleson measures in this case are just the finite measures. But for $n-s p \geq 0$, and $n>1$, the characterization of the Carleson measures for $H_{s}^{p}$ still remains open. In the case where we are "near" the regular case, that is when $n-s p<1$ it is shown in [AhCo], [CohVe1] and [CohVe2], that the Carleson measures for $H_{s}^{p}$ and $K_{s}\left[L^{p}(d \sigma)\right]$ are the same, and any of the different characterizations of the Carleson measures for the last ones also hold for $H_{s}^{p}$.

One of the main purposes of this paper is to extend this situation to $H_{s}^{p}(w)$ for $w$ a weight in $A_{p}$. If $E \subset \mathbf{S}^{n}$ is measurable, we define

$$
W(E)=\int_{E} w d \sigma .
$$


A weight $w$ satisfies a doubling condition of order $\tau$, if there exists $\tau>0$ such that for any nonisotropic ball $B$ in $\mathbf{S}^{n}, W\left(2^{k} B\right) \leq C 2^{k \tau} W(B)$.

It is well known that any weight in $A_{p}$ satisfies a doubling condition of some order $\tau$ strictly less than $n p$. We begin observing that if $\tau-s p<0$, the space $H_{s}^{p}(w)$ consists of continuous functions on $\overline{\mathbf{B}}^{n}$, and consequently, the Carleson measures are just the finite ones. If $\tau-s p<1$, we show that the Carleson measures for $H_{s}^{p}(w)$ and $K_{s}\left[L^{p}(w)\right]$ coincide, whereas if $\tau-s p \geq 1$, this coincidence may fail.

As it happens in the unweighted case (see [CohVe1]), the proof of the characterization of the Carleson measures for $H_{s}^{p}(w)$ will be based in the construction of weighted holomorphic potentials, with control of their $H_{s}^{p}(w)$ norm. In fact, technical reasons give that it is convenient to deal with weighted Triebel-Lizorkin spaces which, on the other hand, have interest on their own. In the second section we study these spaces. If $s \geq 0$, we will write $[s]^{+}$the integer part of $s$ plus 1 . Let $1<p<+\infty, 1 \leq q \leq+\infty$, and $s \geq 0$. The weighted holomorphic Triebel-Lizorkin space $H F_{s}^{p q}(w)$ when $q<+\infty$ is the space of holomorphic functions $f$ in $\mathbf{B}^{n}$ for which

$$
\begin{aligned}
\| f & \|_{H F_{s}^{p q}(w)} \\
& =\left(\int_{\mathbf{S}^{n}}\left(\int_{0}^{1}\left|\left((I+R)^{[s]^{+}} f\right)(r \zeta)\right|^{q}\left(1-r^{2}\right)^{\left([s]^{+}-s\right) q-1} d r\right)^{p / q} w(\zeta) d \sigma(\zeta)\right)^{1 / p} \\
& <+\infty
\end{aligned}
$$

whereas when $q=+\infty$,

$$
\begin{aligned}
& \|f\|_{H F_{s}^{p \infty}(w)} \\
& \quad=\left(\int_{\mathbf{S}^{n}}\left(\sup _{0<r<1}\left|\left((I+R)^{[s]^{+}} f\right)(r \zeta)\right|\left(1-r^{2}\right)^{[s]^{+}-s}\right)^{p} w(\zeta) d \sigma(\zeta)\right)^{1 / p}<+\infty,
\end{aligned}
$$

where $I$ denotes the identity operator.

The Section 2 is devoted to the general theory of weighted holomorphic Triebel-Lizorkin spaces. We give different equivalent definitions of the spaces $H F_{s}^{p q}(w)$ in terms of admissible area functions, we give duality theorems on these spaces, we study some relations of inclusion among them and we also obtain that when $q=2$, the weighted Triebel-Lizorkin space $H F_{s}^{p 2}(w)$ coincides with the weighted Hardy-Sobolev space $H_{s}^{p}(w)$.

The main result in Section 3 is the characterization of the Carleson measures for $H_{s}^{p}(w)$, when $0<\tau-s p<1$, in terms of a positive kernel. 
Theorem C. Let $1<p<+\infty, w$ an $A_{p}$-weight, and $\mu$ a finite positive Borel measure on $\mathbf{B}^{n}$. Assume that $w$ is doubling of order $\tau$, for some $\tau<1+s p$. We then have that the following statements are equivalent:

(i) $\left\|K_{s}(f)\right\|_{L^{p}(d \mu)} \leq C\|f\|_{L^{p}(w)}$.

(ii) $\|f\|_{L^{p}(d \mu)} \leq C\|f\|_{H_{s}^{p}(w)}$.

The proof relies on the construction of weighted holomorphic potentials, with control of their weighted Hardy-Sobolev norm.

We also give examples of the sharpness of the above theorem. We show that if $p=2$ and $\tau>1+s p, n<\tau<n+1$, then there exists $w$ in $A_{2} \cap D_{\tau}$ and a measure $\mu$ on $\mathbf{S}^{n}$ which is Carleson for $H_{s}^{2}(w)$, but it is not Carleson for $K_{s}\left[L^{2}(w)\right]$.

Finally, the usual remark on notation: we will adopt the convention of using the same letter for various absolute constants whose values may change in each occurrence, and we will write $A \preceq B$ if there exists an absolute constant $M$ such that $A \leq M B$. We will say that two quantities $A$ and $B$ are equivalent if both $A \preceq B$ and $B \preceq A$, and, in that case, we will write $A \simeq B$.

\section{§2. Weighted holomorphic Triebel-Lizorkin spaces}

In this section we will introduce weighted holomorphic Triebel-Lizorkin spaces, and we will obtain characterizations in terms of Littlewood-Paley functions and admissible area functions. These characterizations, known in the unweighted case, will be used in the following sections.

We begin recalling some simple facts about $A_{p}$ weights that we will need later. It is well known that $A_{\infty}=\bigcup_{1<p<+\infty} A_{p}$ and that any $A_{p}$ weight satisfies a doubling condition. We recall that a weight $w$ satisfies a doubling condition of order $\tau, \tau>0$, if there exists $C>0$, such that for any nonisotropic ball $B \subset \mathbf{S}^{n}$, and any $k \geq 0, W\left(2^{k} B\right) \leq C 2^{\tau k} W(B)$. We will say that this weight $w$ is in $D_{\tau}$. In fact, if $w \in A_{p}$, there exists $p_{1}<p$ such that $w$ is also in $A_{p_{1}}$, and consequently we have that $w \in D_{\tau}$ for $\tau=n p_{1}<n p$, (see [StrTo]).

Examples of $A_{p}$ weights can be obtained as follows: if $\zeta=\left(\zeta^{\prime}, \zeta_{n}\right)$, and $w(\zeta)=\left(1-\left|\zeta^{\prime}\right|^{2}\right)^{\varepsilon}$, we then have that $w \in A_{p}$ if $-1<\varepsilon<p-1$. We also have that for this weight, $w \in D_{\tau}, \tau=n+\varepsilon$.

The following lemma gives the natural relationships between the spaces $L^{p}(w), w \in A_{p}$, and the Lebesgue spaces $L^{q}(d \sigma)$. 
Lemma 2.1. Let $1<p<+\infty$, and $w$ be an $A_{p}$-weight. We then have:

(i) There exists $1<p_{1}<p$ such that $L^{p}(w) \subset L^{p_{1}}(d \sigma)$.

(ii) There exists $p_{2}>p$ such that $L^{p_{2}}(d \sigma) \subset L^{p}(w)$.

We now proceed to study the weighted holomorphic Triebel-Lizorkin spaces $H_{s}^{p q}(w)$ already defined in the introduction. We begin with some definitions. If $1<q \leq+\infty, k$ an integer such that $k>s \geq 0$, and $\zeta \in \mathbf{S}^{n}$, the Littlewood-Paley type functions are given by

$$
A_{1, k, q, s}(f)(\zeta)=\left(\int_{0}^{1}\left|(I+R)^{k} f(r \zeta)\right|^{q}\left(1-r^{2}\right)^{(k-s) q-1} d r\right)^{1 / q}
$$

when $q<+\infty$, and

$$
A_{1, k, \infty, s}(f)(\zeta)=\sup _{0<r<1}\left|(I+R)^{k} f(r \zeta)\right|\left(1-r^{2}\right)^{k-s}
$$

when $q=+\infty$.

If $\alpha>1, \zeta \in \mathbf{S}^{n}$, we denote by $D_{\alpha}(\zeta), \alpha>1$ the admissible region given by $D_{\alpha}(\zeta)=\left\{z \in \mathbf{B}^{n} ;|1-z \bar{\zeta}|<\alpha(1-|z|)\right\}$. We introduce the admissible area function

$$
A_{\alpha, k, q, s}(f)(\zeta)=\left(\int_{D_{\alpha}(\zeta)}\left|(I+R)^{k} f(z)\right|^{q}\left(1-|z|^{2}\right)^{(k-s) q-n-1} d v(z)\right)^{1 / q}
$$

when $q<+\infty$, where $d v$ is the Lebesgue measure on $\mathbf{B}^{n}$, and in case $q=+\infty$

$$
A_{\alpha, k, \infty, s}(f)(\zeta)=\sup _{z \in D_{\alpha}(\zeta)}\left|(I+R)^{k} f(z)\right|\left(1-|z|^{2}\right)^{k-s}
$$

Our first goal is to obtain that if $1<p<+\infty, 1<q<+\infty$ and $w$ is an $A_{p}$ weight, then an holomorphic function $f$ is in $H F_{s}^{p, q}(w)$ if and only if $A_{\alpha, k, q, s}(f) \in L^{p}(w)$, for some (and then for all) $\alpha \geq 1$ and $k>s$. We will follow the ideas in $[\mathrm{OF}]$. For the sake of completeness, we will sketch the modifications needed to obtain the weighted case.

If $1<p<+\infty, 1<q \leq+\infty$ we denote by

$$
L^{p}(w)\left(L_{1}^{q}\right)=L^{p}(w)\left(L^{q}\left(\frac{2 n r^{2 n-1}}{1-r^{2}} d r\right)\right)
$$


the mixed-norm space of measurable functions $f$ in $\mathbf{S}^{n} \times[0,1]$ such that

$$
\|f\|_{p, q, w}=\left(\int_{\mathbf{S}^{n}}\left(\int_{0}^{1}|f(r \zeta)|^{q} \frac{2 n r^{2 n-1}}{1-r^{2}} d r\right)^{p / q} w(\zeta) d \sigma(\zeta)\right)^{1 / p}<+\infty .
$$

Also if $\alpha>1$, and $E_{\alpha}(z)=\left(\int_{\mathbf{S}^{n}} \chi_{D_{\alpha}(\zeta)}(z) d \sigma(\zeta)\right)^{-1} \simeq\left(1-|z|^{2}\right)^{-n}$, we denote by $L^{p}(w)\left(L_{\alpha}^{q}\right)$ the mixed-norm space of measurable functions $f$ defined in $\mathbf{S}^{n} \times \mathbf{B}^{n}$ such that

$$
\|f\|_{\alpha, p, q, w}=\left(\int_{\mathbf{S}^{n}}\left(\int_{\mathbf{B}^{n}}|f(\zeta, z)|^{q} \frac{E_{\alpha}(z)}{\left(1-|z|^{2}\right)} d v(z)\right)^{p / q} w(\zeta) d \sigma(\zeta)\right)^{1 / p}<+\infty .
$$

We denote by $F^{\alpha, p, q}(w)$ the space of measurable functions on $\mathbf{B}^{n}$ such that

$$
J_{\alpha} f(\zeta, z)=\chi_{D_{\alpha}(\zeta)}(z) f(z)
$$

is in $L^{p}(w)\left(L_{\alpha}^{q}\right)$, normed with the norm induced by $\|\cdot\|_{\alpha, p, q, w}$. We also introduce the space $F^{1, p, q}(w)$ of measurable functions on $\mathbf{B}^{n}$ such that $J_{1} f(\zeta, r)=f(r \zeta)$ is in $L^{p}(w)\left(L_{1}^{q}\right)$.

The representation of the dual of a mixed-norm space, see [BeLo], gives that if $1<p, q<+\infty$, the dual space of $L^{p}(w)\left(L_{1}^{q}\right)$ is $L^{p^{\prime}}(w)\left(L_{1}^{q^{\prime}}\right), 1 / p+$ $1 / p^{\prime}=1,1 / q+1 / q^{\prime}=1$, and that if $f \in F^{1, p, q}(w), g \in F^{1, p^{\prime}, q^{\prime}}(w)$ the pairing is given by

$$
(f, g)=\int_{\mathbf{S}^{n}}\left(\int_{0}^{1} f(r \zeta) \overline{g(r \zeta)} \frac{2 n r^{2 n-1}}{1-r^{2}} d r\right) w(\zeta) d \sigma(\zeta)
$$

Analogously, the dual space of $L^{p}(w)\left(L_{\alpha}^{q}\right)$ is $L^{p^{\prime}}(w)\left(L_{\alpha}^{q^{\prime}}\right)$, and if $f \in$ $F^{\alpha, p, q}(w), g \in F^{\alpha, p^{\prime}, q^{\prime}}(w)$ the pairing is given by

$$
\begin{aligned}
(f, g)_{\alpha} & =\int_{\mathbf{B}^{n}} \int_{\mathbf{S}^{n}} f(z) \overline{g(z)} \chi_{D_{\alpha}(\zeta)}(z) w(\zeta) d \sigma(\zeta) \frac{d v(z)}{\left(1-|z|^{2}\right)^{n+1}} \\
& =\int_{\mathbf{B}^{n}} f(z) \overline{g(z)} \frac{E_{\alpha}^{w}(z)}{\left(1-|z|^{2}\right)^{n+1}} d v(z)
\end{aligned}
$$

where $E_{\alpha}^{w}(z)=\int_{\mathbf{S}^{n}} \chi_{D_{\alpha}(\zeta)}(z) w(\zeta) d \sigma(\zeta)$.

Observe that if we write $z_{0}=z /|z|$, the doubling property of $w$ gives that $E_{\alpha}^{w}(z) \simeq W\left(B\left(z_{0},(1-|z|)\right)\right)$. From now on we will write $B_{z}=$ $B\left(z_{0},(1-|z|)\right)$. 
We begin with two lemmas that are weighted versions of Lemmas 2.2. and 2.3 in $[\mathrm{OF}]$, and whose proofs we omit. We recall that if $\psi$ is a measurable function on $\mathbf{S}^{n}$, the weighted Hardy-Littlewood maximal function is given by

$$
M_{H L}^{w}(\psi)(\zeta)=\sup _{B \ni \zeta} \frac{1}{W(B)} \int_{B}|\psi(\eta)| w(\eta) d \sigma(\eta)
$$

Lemma 2.2. There exist $C>0, N_{0}>0$ such that for any $z \in D_{\alpha}(\zeta)$, $N \geq N_{0}$,

$$
\frac{\left(1-|z|^{2}\right)^{n+N}}{W\left(B_{z}\right)} \int_{\mathbf{S}^{n}} \frac{|\psi(\eta)|}{|1-z \bar{\eta}|^{n+N}} w(\eta) d \sigma(\eta) \leq C M_{H L}^{w}(\psi)(\zeta) .
$$

Lemma 2.3. Let $\alpha>1$. There exists $C>0$, such that for any $z \in$ $D_{\alpha}(\zeta)$,

$$
\frac{1}{W\left(B_{z}\right)} \int_{\mathbf{S}^{n}} \chi_{D_{\alpha}(\eta)}(z)|\psi(\eta)| w(\eta) d \sigma(\eta) \leq C M_{H L}^{w}(\psi)(\zeta)
$$

TheOrem 2.4. Let $1<p<+\infty, 1 \leq q \leq+\infty$, and $\alpha \geq 1$. Then the space $F^{\alpha, p, q}(w)$ is a retract of $L^{p}(w)\left(L_{\alpha}^{q}\right)$.

Proof of Theorem 2.4. The fact that $J_{1}$ is an isometry between $F^{1, p, q}(w)$ and $L^{p}(w)\left(L_{1}^{q}\right)$ gives the theorem for the case $\alpha=1$.

If $\alpha>1$, we introduce the averaging operator

$$
A_{\alpha}(\varphi)(z)=\frac{1}{E_{\alpha}^{w}(z)} \int_{\mathbf{S}^{n}} \chi_{D_{\alpha}(\eta)}(z) \varphi(\eta, z) w(\eta) d \sigma(\eta) .
$$

The definition of $E_{\alpha}^{w}(z)$ gives that $A_{\alpha} \circ J_{\alpha}$ is the identity operator on $F^{\alpha, p, q}(w)$. So, in order to finish the theorem, we need to show that $A_{\alpha}$ maps $L^{p}(w)\left(L_{\alpha}^{q}\right)$ to $F^{\alpha, p, q}(w)$. We consider first the case $1 \leq q \leq p<+\infty$. Let $m=p / q \geq 1$ and let $m^{\prime}$ be the conjugate exponent of $m$. We then have by duality that

$$
\begin{aligned}
& \left\|A_{\alpha}(\varphi)\right\|_{\alpha, p, q, w}^{q} \\
& =\left.\sup _{\|\psi\|_{L^{m^{\prime}(w)}} \leq 1}\left|\int_{\mathbf{S}^{n}} \int_{D_{\alpha}(\zeta)}\right| A_{\alpha}(\varphi)(z)\right|^{q} \frac{d v(z)}{\left(1-|z|^{2}\right)^{n+1}} \psi(\zeta) w(\zeta) d \sigma(\zeta) \mid .
\end{aligned}
$$


Now Hölder's inequality gives that

$$
\left|A_{\alpha}(\varphi)(z)\right|^{q} \leq \frac{1}{E_{\alpha}^{w}(z)} \int_{\mathbf{S}^{n}}|\varphi(\eta, z)|^{q} \chi_{D_{\alpha}(\eta)}(z) w(\eta) d \sigma(\eta) .
$$

Hence, by Lemma 2.3

$$
\begin{aligned}
& \left\|A_{\alpha}(\varphi)\right\|_{\alpha, p, q, w}^{q} \\
& \preceq \sup _{\|\psi\|_{L^{m^{\prime}(w)}} \leq 1} \int_{\mathbf{S}^{n}} \int_{\mathbf{B}^{n}} \frac{1}{E_{\alpha}^{w}(z)} \chi_{D_{\alpha}(\zeta)}(z) \int_{\mathbf{S}^{n}} \chi_{D_{\alpha}(\eta)}(z)|\varphi(\eta, z)|^{q} w(\eta) d \sigma(\eta) \\
& \times \frac{d v(z)}{\left(1-|z|^{2}\right)^{n+1}}|\psi(\zeta)| w(\zeta) d \sigma(\zeta) \\
& \preceq \sup _{\|\psi\|_{L^{m^{\prime}(w)}} \leq 1} \int_{\mathbf{S}^{n}} \int_{\mathbf{B}^{n}}|\varphi(\eta, z)|^{q} \frac{d v(z)}{\left(1-|z|^{2}\right)^{n+1}} w(\eta) M_{H L}^{w}(\psi)(\eta) d \sigma(\eta) .
\end{aligned}
$$

Next, Hölder's inequality with exponent $m=p / q$ gives that the above is bounded by

$$
\begin{aligned}
& \sup _{\|\psi\|_{L^{m^{\prime}(w)}} \leq 1}\left\|M_{H L}^{w} \psi\right\|_{L^{m^{\prime}}(w)} \\
& \quad \times\left(\int_{\mathbf{S}^{n}}\left(\int_{\mathbf{B}^{n}}|\varphi(\eta, z)|^{q} \frac{d v(z)}{\left(1-|z|^{2}\right)^{n+1}}\right)^{p / q} w(\eta) d \sigma(\eta)\right)^{q / p} \\
& \leq \sup _{\|\psi\|_{L^{m^{\prime}(w)}} \leq 1}\|\psi\|_{L^{m^{\prime}}(w)}\|\varphi\|_{\alpha, p, q, w}^{q},
\end{aligned}
$$

where we have used that since $w$ is a doubling measure, the weighted HardyLittlewood maximal function is bounded from $L^{m^{\prime}}(w)$ to $L^{m^{\prime}}(w)$. That finishes the proof of the theorem when $q \leq p$.

So we are lead to deal with the case $1<p<q \leq+\infty$, which can be easily obtained from the previous case using the duality in the mixed-norm spaces $L^{p}(w)\left(L_{\alpha}^{q}\right)$.

This result can be used as in the unweighted case to obtain a characterization of the dual spaces of the weighted spaces $F^{\alpha, p, q}(w)$.

Corollary 2.5. Let $1<p<+\infty, 1<q<+\infty, \alpha>1$, and $w$ an $A_{p}$-weight. Then the dual of $F^{\alpha, p, q}(w)$ is $F^{\alpha, p^{\prime}, q^{\prime}}(w)$ with the pairing given by $(f, g)_{\alpha}$. 
The following proposition will be needed in the proof of the main theorem in this section. If $N>0, M>0$, we consider the operators defined by

$$
P^{N, M} f(y)=\int_{\mathbf{B}^{n}} f(z) \frac{\left(1-|z|^{2}\right)^{N}\left(1-|y|^{2}\right)^{M}}{|1-z \bar{y}|^{n+1+N+M}} d v(z), \quad y \in \mathbf{B}^{n} .
$$

TheOREM 2.6. Let $1<p<+\infty, 1 \leq q<+\infty, \alpha, \beta \geq 1$, and $w$ an $A_{p}$ weight. Then there exists $N_{0}>0$ such that for any $N \geq N_{0}$ and any $M>0$, the operator $P^{N, M}$ is continuous from $F^{\alpha, p, q}(w)$ to $F^{\beta, p, q}(w)$.

Proof of Theorem 2.6. We begin with the case $\alpha, \beta>1$. The case where $1 \leq q \leq p<+\infty$ can be deduced following the scheme of [OF], using Lemma 2.2 .

In the case $1<p<q<+\infty$ we apply duality in the mixed norm space and obtain

$$
\begin{aligned}
& \left\|P^{N, M}(f)\right\|_{\beta, p, q, w}^{q} \\
& \quad=\sup _{\|g\|_{\beta, p^{\prime} q^{\prime}, w} \leq 1}\left|\int_{\mathbf{B}^{n}} P^{N, M}(f)(y) \overline{g(y)} \frac{E_{\beta}^{w}(y)}{\left(1-|y|^{2}\right)^{n+1}} d v(y)\right| \\
& \leq \sup _{\|g\|_{\beta, p^{\prime} q^{\prime}, w} \leq 1}\left(f, \widetilde{P}^{M-1, N+1}(g)\right)_{\alpha},
\end{aligned}
$$

where

$$
\begin{aligned}
& \widetilde{P}^{R, S}(g)(z) \\
& \quad=\int_{\mathbf{B}^{n}} \frac{\left(1-|y|^{2}\right)^{R}\left(1-|z|^{2}\right)^{S} g(y)}{|1-y \bar{z}|^{n+1+R+S}} \frac{E_{\beta}^{w}(y)}{\left(1-|y|^{2}\right)^{n}} \frac{\left(1-|z|^{2}\right)^{n}}{E_{\alpha}^{w}(z)} d v(y) .
\end{aligned}
$$

Observe that when $w \equiv 1$, then $\widetilde{P}^{M, N}(f) \simeq P^{M, N}(f)$. Here we are led to obtain that the operator $\widetilde{P}^{M-1, N+1}$ maps boundedly $F^{\beta, p^{\prime}, q^{\prime}}$ to $F^{\alpha, p^{\prime}, q^{\prime}}$, provided $p<q$. If we claim this proposition, we finish the proof of the theorem. Using (2.1), and applying Hölder's inequality,

$$
\begin{aligned}
\left\|P^{N, M}(f)\right\|_{\beta, p, q, w}^{q} & =\sup _{\|g\|_{\alpha, p^{\prime} q^{\prime}, w} \leq 1}\left(f, \widetilde{P}^{M-1, N-1}(g)\right)_{\alpha} \\
& \leq \sup _{\|g\|_{\alpha, p^{\prime} q^{\prime}, w} \leq 1}\|f\|_{\alpha, p, q, w}\left\|\widetilde{P}^{M-1, N-1}(g)\right\|_{\alpha, p^{\prime}, q^{\prime}, w} \\
& \leq C \sup \|f\|_{\alpha, p, q, w} .
\end{aligned}
$$


The cases $\alpha=1$ and $\beta=1$ are proved in a simmilar way.

To finish the theorem we will prove the claim. Changing the notation, it is enough to prove:

Proposition 2.7. Let $1<q<p<+\infty, \alpha, \beta \geq 1$, and $w$ an $A_{p}$ weight. We then have that there exists $N_{0}>0$ such that for any $N \geq N_{0}$ and any $M \geq 0$,

(i) $\widetilde{P}^{M, N}(1)<+\infty$.

(ii) The operator $\widetilde{P}^{M, N}$ is continuous from $F^{\alpha, p, q}(w)$ to $F^{\beta, p, q}(w)$.

Proof of Proposition 2.7. Let us begin with (i). From the definition of $E_{\alpha}^{w}(z)$ and Fubini's theorem,

$$
\begin{aligned}
& \int_{\mathbf{B}^{n}} \frac{\left(1-|z|^{2}\right)^{M}}{|1-z \bar{y}|^{n+1+M+N}} \frac{E_{\alpha}^{w}(z)}{\left(1-|z|^{2}\right)^{n}} d v(z) \\
& \quad=\int_{\mathbf{S}^{n}} \int_{D_{\alpha}(z)} \frac{\left(1-|z|^{2}\right)^{M}}{|1-z \bar{y}|^{n+1+M+N}} \frac{d v(z)}{\left(1-|z|^{2}\right)^{n}} w(\zeta) d \sigma(\zeta) \\
& \preceq \int_{\mathbf{S}^{n}} \frac{1}{|1-y \bar{\zeta}|^{n+N}} w(\zeta) d \sigma(\zeta),
\end{aligned}
$$

where in last inequality we have used Lemma 2.7 in [OF] since $M>-1$.

Next, let $B_{k}=B\left(y_{0}, 2^{k}\left(1-|y|^{2}\right)\right), k \geq 0$, where $y_{0}=y /|y|$. Since $w$ is doubling and $E_{\alpha}^{w}(y) \simeq W\left(B_{0}\right)$ we have that $W\left(B_{k}\right) \leq C^{k} E_{\alpha}^{w}(y)$. Consequently

$$
\begin{aligned}
& \int_{\mathbf{S}^{n}} \frac{1}{|1-y \bar{\zeta}|^{n+N}} w(\zeta) d \sigma(\zeta) \preceq \sum_{k} \int_{B_{k}} \frac{w(\zeta) d \sigma(\zeta)}{\left(2^{k}\left(1-|y|^{2}\right)\right)^{n+N}} \\
& \quad \preceq \frac{E_{\alpha}^{w}(y)}{\left(1-|y|^{2}\right)^{n+N}} \sum_{k} \frac{C^{k}}{2^{k(n+N)}} \preceq \frac{E_{\alpha}^{w}(y)}{\left(1-|y|^{2}\right)^{n+N}},
\end{aligned}
$$

if $N$ is chosen sufficiently large. That finishes the proof of (i).

Since $m=p / q>1$, duality gives that

$$
\begin{aligned}
& \left\|\widetilde{P}^{M, N}(f)\right\|_{\beta, p, q, w}^{q} \\
& =\left.\sup _{\|\psi\|_{L^{m^{\prime}}(w)} \leq 1}\left|\int_{\mathbf{S}^{n}} \int_{D_{\beta}(\zeta)}\right| \widetilde{P}^{M, N} f(y)\right|^{q} \frac{d v(y)}{\left(1-|y|^{2}\right)^{n+1}} \overline{\psi(\zeta)} w(\zeta) d \sigma(\zeta) \mid .
\end{aligned}
$$


Next, Hölder's inequality shows that if $0<\varepsilon<N$ then

$$
\begin{aligned}
& \left|\widetilde{P}^{M, N}(f)(y)\right|^{q} \\
& \leq \int_{\mathbf{B}^{n}}|f(z)|^{q} \frac{\left(1-|z|^{2}\right)^{M}\left(1-|y|^{2}\right)^{N-\varepsilon}}{|1-z \bar{y}|^{n+1+M+N-\varepsilon}} \frac{E_{\alpha}^{w}(z)}{\left(1-|z|^{2}\right)^{n}} \frac{\left(1-|y|^{2}\right)^{n}}{E_{\alpha}^{w}(y)} d v(z) \\
& \quad \times\left(\int_{\mathbf{B}^{n}} \frac{\left(1-|z|^{2}\right)^{M}\left(1-|y|^{2}\right)^{N+\varepsilon \frac{q^{\prime}}{q}}}{|1-z \bar{y}|^{n+1+M+N+\varepsilon \frac{q^{\prime}}{q}}} \frac{E_{\alpha}^{w}(z)}{\left(1-|z|^{2}\right)^{n}} \frac{\left(1-|y|^{2}\right)^{n}}{E_{\alpha}^{w}(y)} d v(z)\right)^{q / q^{\prime}} \\
& \qquad \int_{\mathbf{B}^{n}}|f(z)|^{q} \frac{\left(1-|z|^{2}\right)^{M}\left(1-|y|^{2}\right)^{N-\varepsilon}}{|1-z \bar{y}|^{n+1+N+M-\varepsilon}} \frac{E_{\alpha}^{w}(z)}{\left(1-|z|^{2}\right)^{n}} \frac{\left(1-|y|^{2}\right)^{n}}{E_{\alpha}^{w}(y)} d v(z),
\end{aligned}
$$

where in last inequality we have used (i).

Consequently,

$$
\begin{aligned}
& \left\|\widetilde{P}^{M, N}(f)\right\|_{\beta, p, q, w}^{q} \\
& \leq C \sup _{\|\psi\|_{L^{m^{\prime}(w)}} \leq 1} \mid \int_{\mathbf{S}^{n}} \int_{y \in D_{\beta}(\zeta)} \int_{\mathbf{B}^{n}} \frac{|f(z)|^{q}\left(1-|z|^{2}\right)^{M}\left(1-|y|^{2}\right)^{N-\varepsilon}}{|1-z \bar{y}|^{n+1+N+M-\varepsilon}} \\
& \times \frac{E_{\alpha}^{w}(z)}{\left(1-|z|^{2}\right)^{n}} \frac{\left(1-|y|^{2}\right)^{n}}{E_{\alpha}^{w}(y)} d v(z) \frac{d v(y)}{\left(1-|y|^{2}\right)^{n+1}} \psi(\zeta) w(\zeta) d \sigma(\zeta) \mid \\
& =C \sup _{\|\psi\|_{L^{m^{\prime}(w)}} \leq 1} \mid \int_{\mathbf{S}^{n}} \int_{\mathbf{B}^{n}} \int_{D_{\beta}(\zeta)} \frac{\left(1-|y|^{2}\right)^{N+n-\varepsilon}}{|1-z \bar{y}|^{n+1+N+M-\varepsilon}} \frac{d v(y)}{E_{\alpha}^{w}(y)\left(1-|y|^{2}\right)^{n+1}} \\
& \times|f(z)|^{q}\left(1-|z|^{2}\right)^{M-n} E_{\alpha}^{w}(z) d v(z) \psi(\zeta) w(\zeta) d \sigma(\zeta) \mid .
\end{aligned}
$$

Next, if $y \in D_{\beta}(\zeta), E_{\alpha}^{w}(y) \simeq W\left(B_{y}\right) \simeq W\left(B\left(\zeta,\left(1-|y|^{2}\right)\right)\right.$, and $|1-z \bar{y}| \simeq$ $\left(1-|y|^{2}\right)+|1-z \bar{\zeta}|$.

Assume first that $|1-z \bar{\zeta}| \leq 1$. Hence,

$$
\begin{gathered}
\int_{D_{\beta}(\zeta)} \frac{\left(1-|y|^{2}\right)^{N+n-\varepsilon}}{|1-z \bar{y}|^{n+1+N+M-\varepsilon}} \frac{d v(y)}{E_{\alpha}^{w}(y)\left(1-|y|^{2}\right)^{n+1}} \\
\simeq \int_{\mathbf{B}^{n}} \frac{\left(1-|y|^{2}\right)^{N-\varepsilon}}{\left(\left(1-|y|^{2}\right)+|1-z \bar{\zeta}|\right)^{n+1+N+M-\varepsilon}} \chi_{D_{\beta}(\zeta)}(y) \\
\quad \times \frac{\left(1-|y|^{2}\right)^{n}}{W\left(B\left(\zeta, 1-|y|^{2}\right)\right)} \frac{d v(y)}{\left(1-|y|^{2}\right)^{n+1}},
\end{gathered}
$$


which by integration in polar coordinates is bounded by

$$
\begin{aligned}
& \int_{0}^{1} \frac{r^{2 n-1}\left(1-r^{2}\right)^{N+n-\varepsilon}}{\left(\left(1-r^{2}\right)+|1-z \bar{\zeta}|\right)^{n+1+N+M-\varepsilon}} \frac{d r}{\left(1-r^{2}\right) W\left(B\left(\zeta, C\left(1-r^{2}\right)\right)\right)} \\
& \simeq \int_{0}^{|1-z \bar{\zeta}|} \frac{t^{N+n-\varepsilon-1}}{(t+|1-z \bar{\zeta}|)^{n+1+N+M-\varepsilon}} \frac{d t}{W(B(\zeta, t))} \\
& \quad+\int_{|1-z \bar{\zeta}|}^{1} \frac{t^{N+n-\varepsilon-1}}{(t+|1-z \bar{\zeta}|)^{n+1+N+M-\varepsilon}} \frac{d t}{W(B(\zeta, t))}=I+I I .
\end{aligned}
$$

In $I$ we have that $(t+|1-z \bar{\zeta}|) \simeq|1-z \bar{\zeta}|$, and, since $w \in A_{p}$,

$$
\frac{t^{n}}{W(B(\zeta, t))} \simeq\left(\frac{1}{t^{n}} \int_{B(\zeta, t)} w^{-\left(p^{\prime}-1\right)}\right)^{p-1} .
$$

Thus we obtain that

$$
\begin{aligned}
I & \simeq \int_{0}^{|1-z \bar{\zeta}|} \frac{t^{N-\varepsilon-1}}{|1-z \bar{\zeta}|^{n+1+N+M-\varepsilon}}\left(\frac{1}{t^{n}} \int_{B(\zeta, t)} w^{-\left(p^{\prime}-1\right)}\right)^{p-1} d t \\
& \preceq\left(\int_{B(\zeta,|1-z \bar{\zeta}|)} w^{-\left(p^{\prime}-1\right)}\right)^{p-1} \frac{1}{|1-z \bar{\zeta}|^{n+1+N+M-\varepsilon}} \int_{0}^{|1-z \bar{\zeta}|} t^{N-\varepsilon-n\left(p^{\prime}-1\right)-1} d t \\
& \preceq \frac{1}{|1-z \bar{\zeta}|^{M+1}} \frac{1}{W\left(B\left(z_{0},|1-z \bar{\zeta}|\right)\right)},
\end{aligned}
$$

where we have used that $N>0$ is chosen big enough, and that $w$ satisfies the $A_{p}$ condition.

In $I I,(t+|1-z \bar{\zeta}|) \simeq t$, and since $M+1>0$, we have

$$
\begin{aligned}
I I & \simeq \int_{|1-z \bar{\zeta}|}^{1} \frac{1}{t^{M+2}} \frac{d t}{W(B(\zeta, t))} \leq \int_{|1-z \bar{\zeta}|}^{1} \frac{1}{t^{M+2}} \frac{d t}{W(B(\zeta,|1-z \bar{\zeta}|))} \\
& \preceq \frac{1}{|1-z \bar{\zeta}|^{M+1}} \frac{1}{W\left(B\left(z_{0},|1-z \bar{\zeta}|\right)\right)} .
\end{aligned}
$$

If $|1-z \bar{\zeta}|>1$, then we have that $\left(1-r^{2}\right)+|1-z \bar{\zeta}| \simeq 1$. We return to $(2.5)$ and obtain

$$
\begin{aligned}
& \int_{0}^{1} \frac{\left(1-r^{2}\right)^{N+n-\varepsilon-1} d r}{\left((1-r)^{2}+|1-z \bar{\zeta}|\right)^{n+1+N+M-\varepsilon} W\left(B\left(\zeta, 1-r^{2}\right)\right)} \\
& \quad \preceq\left(\int_{B(\zeta, 1)} w^{-p^{\prime} / p}\right)^{p / p^{\prime}} \int_{0}^{1} t^{N-\varepsilon-n \frac{p}{p^{\prime}-1}} d t \\
& \preceq \frac{1}{|1-z \bar{\zeta}|^{M+1}} \frac{1}{W\left(B\left(z_{0},|1-z \bar{\zeta}|\right)\right)} .
\end{aligned}
$$


Then we have in any case that (2.5) is bounded by

$$
\frac{1}{|1-z \bar{\zeta}|^{M+1}} \frac{1}{W\left(B\left(z_{0},|1-z \bar{\zeta}|\right)\right)} .
$$

In consequence, we return to (2.4) and we obtain

$$
\begin{aligned}
& \left\|\widetilde{P}^{M, N}(f)\right\|_{\beta, p, q, w}^{q} \\
& \left.\preceq \sup _{\|\psi\|_{L^{m^{\prime}(w)}} \leq 1}\left|\int_{\mathbf{S}^{n}} \int_{\mathbf{B}^{n}}\right| f(z)\right|^{q} \frac{\left(1-|z|^{2}\right)^{M-n} E_{\alpha}^{w}(z)}{|1-z \bar{\zeta}|^{M+1} W\left(B\left(z_{0},|1-z \bar{\zeta}|\right)\right)} \\
& \quad \times \psi(\zeta) d v(z) w(\zeta) d \sigma(\zeta) \mid \\
& \left.\preceq \sup _{\|\psi\|_{L^{m^{\prime}(w)}} \leq 1}\left|\int_{\mathbf{S}^{n}} \int_{\mathbf{B}^{n}}\right| f(z)\right|^{q}\left(1-|z|^{2}\right)^{M-n} \chi_{D_{\alpha}(\eta)}(z) \\
& \quad \times \int_{\mathbf{S}^{n}} \frac{\psi(\zeta) w(\zeta) d \sigma(\zeta)}{|1-z \bar{\zeta}|^{M+1} W\left(B\left(z_{0},|1-z \bar{\zeta}|\right)\right)} d v(z) w(\eta) d \sigma(\eta) \mid .
\end{aligned}
$$

Next, if $z \in D_{\alpha}(\eta), B(\eta,|1-z \bar{\zeta}|) \subset B\left(z_{0}, C|1-z \bar{\zeta}|\right)$, and if $B_{k}=B\left(\eta, 2^{k}(1-\right.$ $\left.\left.|z|^{2}\right)\right), k \geq 0$ and $\zeta \in B_{k+1} \backslash B_{k},|1-z \bar{\zeta}| \simeq 2^{k}\left(1-|z|^{2}\right)$. Thus

$$
\begin{aligned}
& \int_{\mathbf{S}^{n}} \frac{|\psi(\zeta)| w(\zeta) d \sigma(\zeta)}{|1-z \bar{\zeta}|^{M+1} W\left(B\left(z_{0},|1-z \bar{\zeta}|\right)\right)} \\
& \qquad \frac{1}{\left(1-|z|^{2}\right)^{M+1} W\left(B\left(\eta, 1-|z|^{2}\right)\right)} \int_{B_{0}}|\psi(\zeta)| w(\zeta) d \sigma(\zeta) \\
& \quad+\sum_{k \geq 1} \frac{1}{2^{k(M+1)}\left(1-|z|^{2}\right)^{M+1} W\left(B\left(\eta, 2^{k}\left(1-|z|^{2}\right)\right)\right)} \int_{B_{k}}|\psi(\zeta)| w(\zeta) d \sigma(\zeta) \\
& \preceq \frac{1}{\left(1-|z|^{2}\right)^{M+1}} \sum_{k \geq 0} \frac{1}{2^{k(M+1)}} M_{H L}^{w}(\psi)(\eta) \preceq \frac{1}{\left(1-|z|^{2}\right)^{M+1}} M_{H L}^{w}(\psi)(\eta) .
\end{aligned}
$$

Plugging the above estimate in (2.6) and using Hölder's inequality with exponent $m=p / q$, we obtain

$$
\begin{aligned}
\left\|\widetilde{P}^{M, N}(f)\right\|_{\beta, p, q, w}^{q} \preceq \sup _{\psi \in L^{m^{\prime}(w)}} \int_{\mathbf{S}^{n}} \int_{\mathbf{B}^{n}}|f(z)|^{q} \frac{1}{\left(1-|z|^{2}\right)^{n+1}} \chi_{D_{\alpha}(\eta)}(z) d v(z) \\
\quad \times M_{H L}^{w}(\psi)(\eta) w(\eta) d \sigma(\eta) \\
\\
\preceq \sup _{\psi \in L^{m^{\prime}(w)}}\|f\|_{\alpha, p, q, w}^{q}\left\|M_{H L}^{w}(\psi)\right\|_{L^{m^{\prime}(w)}}^{q} \preceq\|f\|_{\alpha, p, q, w}^{q} .
\end{aligned}
$$


We deduce from the previous theorem the following characterization of the weighted holomorphic Triebel-Lizorkin spaces. If $f \in H\left(\mathbf{B}^{n}\right), s, t>0$, let

$$
L_{s}^{t} f(z)=\left(1-|z|^{2}\right)^{t-s}(I+R)^{t} f(z) .
$$

Theorem 2.8. Let $1<p<+\infty, 1<q<+\infty, t>s \geq 0$ and $\alpha \geq 1$. Let

$$
H F_{s}^{\alpha, t, p, q}(w)=\left\{f \in H\left(\mathbf{B}^{n}\right) ;\left\|L_{s}^{t} f\right\|_{\alpha, p, q}<+\infty\right\} .
$$

Then $H F_{s}^{\alpha, t, p, q}(w)=H F_{s}^{p q}(w)$.

Proof of Theorem 2.8. If $s<t_{0}<t_{1}, \alpha, \beta \geq 1$, we just need to check that $H F_{s}^{\alpha, t_{0}, p, q}(w)=H F_{s}^{\beta, t_{1}, p, q}(w)$. Any holomorphic function $f$ on $\mathbf{B}^{n}$ satisfying that $L_{s}^{t} f(z) \in F^{\alpha, p, q}(w)$ is in $A^{-\infty}\left(\mathbf{B}^{n}\right)$, the space of holomorphic functions in $\mathbf{B}^{n}$ for which there exists $k>0$ such that $\sup _{z}\left(1-|z|^{2}\right)^{k}|f(z)|<$ $+\infty$. Consequently, $f$ and its derivatives have a representation formula via the reproducing kernel $c_{N} \frac{\left(1-|z|^{2}\right)^{N}}{(1-\bar{z} y)^{n+1+N}}$, for $N>0$ sufficiently large and an adequate constant $c_{N}$. Once we have made this observation, we can reproduce the arguments in $[\mathrm{OF}]$ and obtain

$$
(I+R)^{t_{0}} f(y)=C_{N} \int_{\mathbf{B}^{n}}(I+R)^{t_{1}} f(z)\left(I+R_{y}\right)^{t_{0}-t_{1}} \frac{\left(1-|z|^{2}\right)^{N}}{(1-y \bar{z})^{n+1+N}} d v(z) .
$$

Since for $m>0$ we have that

$$
(I+R)^{-m} g(y)=\frac{1}{\Gamma(m)} \int_{0}^{1}\left(\log \frac{1}{r}\right)^{m-1} g(r y) d r
$$

we obtain

$$
\begin{aligned}
\left\|L_{s}^{t_{0}} f\right\|_{\alpha, p, q, w} & \preceq\left\|\int_{\mathbf{B}^{n}}\left|(I+R)^{t_{1}} f(z)\right| \frac{\left(1-|z|^{2}\right)^{N}\left(1-|y|^{2}\right)^{t_{0}-s}}{|1-\bar{z} y|^{n+1+N+t_{0}-t_{1}}} d v(z)\right\|_{\alpha, p, q, w} \\
& =\left\|P^{N-t_{1}+s, t_{0}-s}\left(\left|L_{s}^{t_{1}} f\right|\right)\right\|_{\alpha, p, q, w}
\end{aligned}
$$

and we just have to apply Theorem 2.6 to finish the proof.

TheOrem 2.9. Let $1<p<+\infty, 1<q<+\infty$, w an $A_{p}$-weight, and $f$ a holomorphic function. Then the following assertions are equivalent:

(i) $f$ is in $H F_{s}^{p q}(w)$.

(ii) $A_{\alpha, k, q, s}(f) \in L^{p}(w)$, for some $\alpha \geq 1$ and $k>s$.

(iii) $A_{\alpha, k, q, s}(f) \in L^{p}(w)$, for all $\alpha \geq 1$ and $k>s$. 
Our next result studies some inclusion relationships between different weighted holomorphic Triebel-Lizorkin spaces.

TheOREM 2.10. Let $1<p<+\infty, 1 \leq q_{0} \leq q_{1} \leq+\infty, s \geq 0$ and let $w$ be an $A_{p}$-weight. We then have

$$
H F_{s}^{p q_{0}}(w) \subset H F_{s}^{p q_{1}}(w)
$$

Proof of Theorem 2.10. We begin with the case $q_{1}=+\infty$. Let $0<$ $\varepsilon<1$. If $L_{s}^{k} f(z)=\left(1-|z|^{2}\right)^{k-s}(I+R)^{k} f(z)$, the fact that $(I+R)^{k} f$ is holomorphic gives that

$$
\left|L_{s}^{k} f(r \zeta)\right| \preceq\left(\frac{1}{\left(1-r^{2}\right)^{n+1}} \int_{K\left(r \zeta, c\left(1-r^{2}\right)\right)}\left|(I+R)^{k} f(z)\right|^{\varepsilon} d v(z)\right)^{1 / \varepsilon}\left(1-r^{2}\right)^{k-s}
$$

where for $y \in \mathbf{B}^{n} K(y, t)$ is the nonisotropic ball in $\mathbf{B}^{n}$ given by

$$
K(y, t)=\left\{z \in \mathbf{B}^{n} ;|\bar{z}(z-y)|+|\bar{y}(y-z)|<t\right\}
$$

In $[\mathrm{OF}]$ it is obtained that

$$
\left|L_{s}^{k} f(r \zeta)\right| \preceq\left(M_{H L}\left(\int_{0}^{1}\left|(I+R)^{k} f(t \eta)\right|^{q}\left(1-t^{2}\right)^{(k-s) q-1} d t\right)^{\varepsilon / q}(\zeta)\right)^{1 / \varepsilon}
$$

Thus

$$
\begin{aligned}
& \|f\|_{H F_{s}^{p \infty}(w)}^{p}=\int_{\mathbf{S}^{n}} \sup _{0<r<1}\left|L_{s}^{k} f(r \zeta)\right|^{p} w(\zeta) d \sigma(\zeta) \\
& \preceq \int_{\mathbf{S}^{n}}\left(M_{H L}\left(\int_{0}^{1}\left|(I+R)^{k} f(t \eta)\right|^{q}\left(1-t^{2}\right)^{(k-s) q-1} d t\right)^{\varepsilon / q}(\zeta)\right)^{p / \varepsilon} \\
& \quad \times w(\zeta) d \sigma(\zeta) .
\end{aligned}
$$

Since $p / \varepsilon>p$, and $w$ is an $A_{p}$-weight, $w$ is in $A_{p / \varepsilon}$, and in consequence the unweighted Hardy-Littlewood maximal function is a bounded map $L^{p / \varepsilon}(w)$ to itself. Hence the above is bounded by

$$
\begin{aligned}
& C \int_{\mathbf{S}^{n}}\left(\int_{0}^{1}\left|(I+R)^{k} f(t \zeta)\right|^{q}\left(1-t^{2}\right)^{(k-s) q-1} d t\right)^{p / q} w(\zeta) d \sigma(\zeta) \\
& =C\|f\|_{H F_{s}^{p q}(w)}^{p}
\end{aligned}
$$


In order to finish the theorem, we will prove that if $q_{0}<q_{1}<+\infty$, then

$$
\|f\|_{H F_{s}^{p q_{1}}(w)} \leq\|f\|_{H F_{s}^{p q_{0}}(w)}^{\frac{q_{0}}{q_{1}}}\|f\|_{H F_{s}^{p \infty}(w)}^{1-\frac{q_{0}}{q_{1}}} .
$$

Since

$$
\begin{aligned}
\|f\|_{H F_{s}^{p q_{1}}(w)}^{p} & \leq \int_{\mathbf{S}^{n}}\left(\sup _{0<r<1}\left|(I+R)^{k} f(r \zeta)\right|(1-r)^{k-s}\right)^{\left(q_{1}-q_{0}\right) p / q_{1}} \\
& \times\left(\int_{0}^{1}\left|(I+R)^{k} f(r \zeta)\right|^{q_{0}}\left(1-r^{2}\right)^{(k-s) q_{0}-1} d r\right)^{p / q_{1}} w(\zeta) d \sigma(\zeta)
\end{aligned}
$$

Hölder's inequality with exponent $q_{1} / q_{0}>1$, gives that the above is bounded by

$$
C\|f\|_{H F_{s}^{p q_{0}(w)}}^{p \frac{q_{0}}{q_{1}}}\|f\|_{H F_{s}^{p \infty}(w)}^{p\left(1-\frac{q_{0}}{q_{1}}\right)}
$$

We now consider the weighted Hardy space $H^{p}(w)$, for $1<p<+\infty$, and $w$ an $A_{p}$ weight. It is shown in [Lu] that $f \in H^{p}(w)$ if and only if $f=C\left[f^{*}\right]$, where $f^{*}(\zeta)=\lim _{r \rightarrow 1} f(r \zeta) \in L^{p}(w)$ is the radial limit, $C$ is the Cauchy-Szegö kernel. In addition, $f=P\left[f^{*}\right]$, where $P$ is the Poisson-Szegö kernel. It follows also that $\|f\|_{H^{p}(w)}^{p} \simeq\left\|f^{*}\right\|_{L^{p}(w)}$.

It is immediate to deduce from this that $f \in H^{p}(w)$ if and only if for any $\alpha \geq 1, M_{\alpha}(f) \in L^{p}(w)$, where $M_{\alpha}$ is the $\alpha$-admissible maximal operator given by

$$
M_{\alpha}(f)(\zeta)=\sup _{z \in D_{\alpha}(\zeta)}|f(z)|
$$

In addition $\|f\|_{H^{p}(w)} \simeq\left\|M_{\alpha}(f)\right\|_{L^{p}(w)}$, with constant that depends on $\alpha$. Indeed, since $|f(r \zeta)| \leq M_{\alpha}(f)(\zeta)$, we have that $\|f\|_{H^{p}(w)} \leq\left\|M_{\alpha}(f)\right\|_{L^{p}(w)}$. On the other hand, assume that $f \in H^{p}(w)$. Then $f=P\left[f^{*}\right], f^{*} \in L^{p}(w)$ and since $M_{\alpha}(f) \leq C M_{H L}\left(f^{*}\right)$, (see for instance [Ru]), we deduce that

$$
\begin{aligned}
\int_{\mathbf{S}^{n}}\left(M_{\alpha}(f)(\zeta)\right)^{p} w(\zeta) d \sigma(\zeta) & \preceq \int_{\mathbf{S}^{n}}\left(M_{H L}\left(f^{*}\right)(\zeta)\right)^{p} w(\zeta) d \sigma(\zeta) \\
& \preceq \int_{\mathbf{S}^{n}}\left|f^{*}(\zeta)\right|^{p} w(\zeta) d \sigma(\zeta) \preceq\|f\|_{H^{p}(w)}^{p}
\end{aligned}
$$

where we have used that since $w$ in an $A_{p}$-weight, the Hardy-Littlewood maximal operator maps $L^{p}(w)$ continuously to itself.

Our next result gives a proof for the weighted nonisotropic case of the fact that the spaces $H^{p}(w)$ can also be defined in terms of admissible area 
functions. Similar results, but using a different approach based on localized good-lambda inequalities, have been obtained in [StrTo] for weighted isotropic Hardy spaces in $\mathbf{R}^{n}$.

TheOrem 2.11. Let $1<p<+\infty$, and $w$ be an $A_{p}$-weight. Let $f$ be an holomorphic function on $\mathbf{B}^{n}$. Then the following assertions are equivalent:

(i) $f$ is in $H^{p}(w)$.

(ii) There exists $\alpha \geq 1, k>0$, such that $A_{\alpha, k, 2,0}(f) \in L^{p}(w)$.

(iii) For every $\alpha \geq 1$, and $k>0, A_{\alpha, k, 2,0}(f) \in L^{p}(w)$.

In addition, there exists $C>0$ such that for any $f \in H^{p}(w)$,

$$
\frac{1}{C}\|f\|_{H^{p}(w)} \leq\left\|A_{\alpha, 1,2,0}(f)\right\|_{L^{p}(w)} \leq C\|f\|_{H^{p}(w)} .
$$

Proof of Theorem 2.11. We already know that (ii) and (iii) are equivalent, so we only have to check the equivalence of (i) and (ii) for the case $k=1$. The proof of (i) implies (ii) is given in [KaKo], using the arguments of [St2]. For the proof of (ii) implies (i), we will follow some ideas of [AhBrCa].

Without loss of generality we may assume that $f(0)=0$. Let us assume first that $f \in H\left(\overline{\mathbf{B}^{n}}\right)$. Then $f=P\left[f^{*}\right]$ where $f^{*} \in \mathcal{C}\left(\mathbf{S}^{n}\right)$. We want to check that

$$
\left\|f^{*}\right\|_{L^{p}(w)} \leq C\left\|A_{\alpha, 1,2,0}(f)\right\|_{L^{p}(w)} .
$$

We will use that the dual space of $L^{p}(w)$ can be identified with $L^{p^{\prime}}\left(w^{-\left(p^{\prime}-1\right)}\right)$ if the duality is given by

$$
\langle f, g\rangle=\int_{\mathbf{S}^{n}} f(\zeta) \overline{g(\zeta)} d \sigma(\zeta)
$$

Hence,

$$
\left\|f^{*}\right\|_{L^{p}(w)}=\sup \left\{\left|\int_{\mathbf{S}^{n}} f^{*}(\zeta) g^{*}(\zeta) d \sigma(\zeta)\right|, g^{*} \in \mathcal{C}\left(\mathbf{S}^{n}\right),\left\|g^{*}\right\|_{L^{p^{\prime}}\left(w^{-\left(p^{\prime}-1\right)}\right)} \leq 1\right\} .
$$

If $g=P\left[g^{*}\right]$, we have (see $[\mathrm{AhBrCa}]$ page 131 )

$$
\begin{aligned}
& \frac{n \pi^{n}}{(n-1) !} \int_{\mathbf{S}^{n}} f^{*}(\zeta) g^{*}(\zeta) d \sigma(\zeta) \\
& =n^{2} \int_{\mathbf{B}^{n}} f(z) g(z) d v(z)+\int_{\mathbf{B}^{n}}\left(\nabla_{\mathbf{B}^{n}} f(z), \nabla_{\mathbf{B}^{n}} g(z)\right)_{\mathbf{B}^{n}} \frac{d v(z)}{1-|z|^{2}}
\end{aligned}
$$


where $\nabla_{\mathbf{B}^{n}}$ is the gradient in the Bergman metric (see for instance [St2]), and

$$
(F(z), G(z))_{\mathbf{B}^{n}}=\left(1-|z|^{2}\right)\left(\sum_{i, j}\left(\delta_{i, j}-z_{i} \overline{z_{j}}\right) F_{i}(z) \bar{G}_{j}(z)\right) .
$$

We then have (see $[\mathrm{St2}]$ ) that since $F$ is holomorphic

$$
\begin{aligned}
\left\|\nabla_{\mathbf{B}^{n}} F(z)\right\|_{\mathbf{B}^{n}}^{2} & =\left(\nabla_{\mathbf{B}^{n}} F(z), \nabla_{\mathbf{B}^{n}} F(z)\right)_{\mathbf{B}^{n}} \\
& \simeq\left(1-|z|^{2}\right)\left\{\sum_{i=1}^{n}\left|\frac{\partial}{\partial z_{i}} F(z)\right|^{2}-\left|\sum_{i=1}^{n} z_{i} \frac{\partial}{\partial z_{i}} F(z)\right|^{2}\right\} .
\end{aligned}
$$

In order to estimate $\int_{\mathbf{B}^{n}} f(z) g(z) d v(z)$ we will need to obtain estimates of the values of the functions $f, g$ on compact subsets of $\mathbf{B}^{n}$ in terms of the norms $\left\|A_{\alpha, 1,2,0}(f)\right\|_{L^{p}(w)}$ and $\left\|A_{\alpha, 1,2,0}(g)\right\|_{L^{p^{\prime}\left(w^{-\left(p^{\prime}-1\right)}\right)}}$ respectively.

Lemma 2.12. Let $1<p<+\infty$ and $w$ an $A_{p}$-weight. There exists $C>0$ such that for any holomorphic function $f$ in $\mathbf{B}^{n}$, and any $z=r \zeta$

$$
|f(z)| \preceq\left(|f(0)|+\int_{0}^{r} \frac{d t}{W\left(B\left(\zeta, 1-t^{2}\right)\right)^{1 / p}\left(1-t^{2}\right)}\left\|A_{\alpha, 1,2,0}(f)\right\|_{L^{p}(w)}^{p}\right) .
$$

In particular, if $K \subset \mathbf{B}^{n}$ is compact and

$$
\|f\|_{K}=\sup _{z \in K}|f(z)|,
$$

then there exists a constant $C>0$, depending only on $w, p$ and $K$ such that $\|f\|_{K} \leq C\left(|f(0)|+\left\|A_{\alpha, 1,2,0}(f)\right\|_{L^{p}(w)}\right)$.

Proof of Lemma 2.12. Since $f$ is holomorphic, we obtain that if $z=$ $r \zeta \in \mathbf{B}^{n}$, there exist $C_{i}>0, i=1,2$, such that for any $\eta \in B\left(\zeta, C_{1}\left(1-r^{2}\right)\right)$, then

$$
\begin{aligned}
|\nabla f(z)|^{2} & \preceq \frac{1}{\left(1-|z|^{2}\right)^{n+1}} \int_{K\left(z, C_{2}\left(1-|z|^{2}\right)\right)}|\nabla f(y)|^{2} d v(y) \\
& \preceq \frac{1}{\left(1-|z|^{2}\right)^{2}} \int_{K\left(z, C_{2}\left(1-|z|^{2}\right)\right)}\left(1-|y|^{2}\right)^{1-n}|\nabla f(y)|^{2} d v(y) \\
& \leq \frac{C}{\left(1-|z|^{2}\right)^{2}}\left(A_{\alpha, 1,2,0}(f)(\eta)\right)^{2}
\end{aligned}
$$

Consequently

$$
\left(\left(1-|z|^{2}\right)|\nabla f(z)|\right)^{p} \preceq\left(A_{\alpha, 1,2,0}(f)(\eta)\right)^{p} .
$$


Then we have

$$
\begin{aligned}
& \left(\left(1-|z|^{2}\right)|\nabla f(z)|\right)^{p} W\left(B\left(\zeta, 1-r^{2}\right)\right) \\
& \quad \preceq \int_{B\left(\zeta, 1-r^{2}\right)}\left(A_{\alpha, 1,2,0}(f)(\eta)\right)^{p} w(\eta) d \sigma(\eta) \preceq\left\|A_{\alpha, 1,2,0}(f)\right\|_{L^{p}(w)}^{p}
\end{aligned}
$$

In particular, if $0<r<1$ and $\zeta \in \mathbf{S}^{n}$,

$$
\left|\frac{\partial f}{\partial r}(r \zeta)\right| \preceq \frac{1}{W\left(B\left(\zeta, 1-r^{2}\right)\right)^{1 / p}\left(1-r^{2}\right)}\left\|A_{\alpha, 1,2,0}(f)\right\|_{L^{p}(w)},
$$

and integrating, we finally obtain

$$
|f(r \zeta)| \preceq\left(|f(0)|+\int_{0}^{r} \frac{d t}{W\left(B\left(\zeta, 1-t^{2}\right)\right)^{1 / p}\left(1-t^{2}\right)}\left\|A_{\alpha, 1,2,0}(f)\right\|_{L^{p}(w)}\right) .
$$

For the remaining affirmation, let $K \subset \mathbf{B}^{n}$ be compact. Then there exists $0<\delta<1$ such that for any $z=r \zeta \in K, r \leq 1-\delta$, and

$$
|f(z)| \preceq\left(|f(0)|+\frac{1}{W(B(\zeta, \delta))^{1 / p} \delta}\left\|A_{\alpha, 1,2,0}(f)\right\|_{L^{p}(w)}\right) .
$$

Since $w$ is doubling, and there exists $N>0$ (not depending on $\zeta$ ) such that $\left.\mathbf{S}^{n} \subset B(\zeta, c N \delta)\right), W\left(\mathbf{S}^{n}\right) \preceq W(B(\zeta, \delta))$, and consequently

$$
\|f\|_{K} \preceq|f(0)|+\left\|A_{\alpha, 1,2,0}(f)\right\|_{L^{p}(w)} .
$$

Going back to the proof of the Theorem 2.11, let $0<\varepsilon<1$. The above lemma together with the fact that if $w$ is an $A_{p}$ weight, then $w^{-\left(p^{\prime}-1\right)}$ is an $A_{p^{\prime}}$-weight, give by (2.8) that

$$
\begin{aligned}
& \left|\int_{\mathbf{S}^{n}} f^{*}(\zeta) g^{*}(\zeta) d \sigma(\zeta)\right| \preceq\left\|A_{\alpha, 1,2,0}(f)\right\|_{L^{p}(w)}\left\|A_{\alpha, 1,2,0}(g)\right\|_{L^{p^{\prime}\left(w^{-\left(p^{\prime}-1\right)}\right)}} \\
& \quad+\left|\int_{1-\varepsilon \leq|z|<1} f(z) g(z) d v(z)\right|+\int_{\mathbf{B}^{n}}\left\|\nabla_{\mathbf{B}^{n}} f(z)\right\|_{\mathbf{B}^{n}}\left\|\nabla_{\mathbf{B}^{n}} g(z)\right\|_{\mathbf{B}^{n}} \frac{d v(z)}{1-|z|^{2}} .
\end{aligned}
$$

In order to estimate the second integral, we use polar coordinates, and obtain

$$
\left|\int_{1-\varepsilon \leq|z|<1} f(z) g(z) d v(z)\right|
$$


which by Hölder's inequality is bounded by

$$
\begin{aligned}
& \int_{1-\varepsilon}^{1} \int_{\mathbf{S}^{n}}\left|f(r \zeta)\left\|g(r \zeta) \mid d \sigma(\zeta) d r \preceq \int_{1-\varepsilon}^{1}\right\| f_{r}\left\|_{L^{p}(w)}\right\| g_{r} \|_{L^{p^{\prime}\left(w^{-\left(p^{\prime}-1\right)}\right)}} d r\right.
\end{aligned}
$$

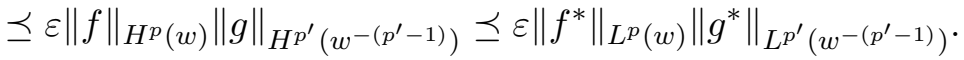

For the third integral, we use (5.1) of [CoiMeSt] to estimate it by

$$
\begin{aligned}
& \int_{\mathbf{S}^{n}} A_{\alpha, 1,2,0}(f)(\zeta) A_{\alpha, 1,2,0}(g)(\zeta) d \sigma(\zeta) \\
& \quad \preceq\left\|A_{\alpha, 1,2,0}(f)\right\|_{L^{p}(w)}\left\|A_{\alpha, 1,2,0}(g)\right\|_{L^{p^{\prime}}\left(w^{-\left(p^{\prime}-1\right)}\right)}
\end{aligned}
$$

Since we already know (see $[\mathrm{KaKo}])$ that $\left\|A_{\alpha, 1,2,0}(g)\right\|_{L^{p^{\prime}}\left(w^{-\left(p^{\prime}-1\right)}\right)} \preceq$ $\left\|g^{*}\right\|_{L^{p^{\prime}}\left(w^{-\left(p^{\prime}-1\right)}\right)}$, we finally obtain

$$
\left\|f^{*}\right\|_{L^{p}(w)} \preceq\left\|A_{\alpha, 1,2,0}(f)\right\|_{L^{p}(w)}+\varepsilon\left\|f^{*}\right\|_{L^{p}(w)},
$$

which gives the result for $f \in H\left(\overline{\mathbf{B}}^{n}\right)$.

So we are left to show that the estimate we have already obtained holds for a general holomorphic function in $\mathbf{B}^{n}$. If $f$ is an holomorphic function on $\mathbf{B}^{n}$ such that $\left\|A_{\alpha, 1,2,0}(f)\right\|_{L^{p}(w)}<+\infty$, let $f_{r}(z)=f(r z) \in H\left(\overline{\mathbf{B}^{n}}\right)$, for $0<r<1$. We then have that

$$
\left\|f_{r}\right\|_{H^{p}(w)} \preceq\left\|A_{\alpha, 1,2,0}\left(f_{r}\right)\right\|_{L^{p}(w)} .
$$

Let us check first that

$$
\sup _{r}\left\|A_{\alpha, 1,2,0}\left(f_{r}\right)\right\|_{L^{p}(w)} \leq C\left\|A_{\alpha, 1,2,0}(f)\right\|_{L^{p}(w)} .
$$

Notice that

$$
\left\|A_{\alpha, 1,2,0}\left(f_{r}\right)\right\|_{L^{p}(w)}^{p}=\left\|J_{\alpha}\left(\left(1-|\cdot|^{2}\right)(I+R) f_{r}\right)\right\|_{L^{p}(w)\left(L^{2}\left(\frac{d v(z)}{(1-|z| 2)^{n+1}}\right)\right)} .
$$

We will check that there exists $0 \leq G(\zeta, z) \in L^{p}(w)\left(L^{2}\left(\frac{d v(z)}{\left(1-|z|^{2}\right)^{n+1}}\right)\right)$ such that for any $0<r<1, \zeta \in \mathbf{S}^{n}, z \in \mathbf{B}^{n}, J_{\alpha}\left(\left(1-|\cdot|^{2}\right)(I+R) f_{r}\right)(\zeta, z) \leq$ $G(\zeta, z)$, and $\|G\|_{L^{p}(w)\left(L^{2}\left(\frac{d v(z)}{\left(1-|z|^{2}\right)^{n+1}}\right)\right)} \preceq\left\|A_{\alpha, 1,2,0}(f)\right\|_{L^{p}(w)} \cdot$

Let us obtain such a function $G$. Since by hypothesis $A_{\alpha, 1,2,0} f \in L^{p}(w)$, we have that the holomorphic function $f$ satisfies that $A_{\alpha, 1,2,0} f \in L^{1}(d \sigma)$, and consequently that there exists $C>0$ such that for any $z \in \mathbf{B}^{n},|f(z)| \preceq$ 
$1 /\left(1-|z|^{2}\right)^{n}$. Hence, the integral representation theorem gives that for $N>0$ sufficiently large, and $z \in \mathbf{B}^{n}$,

$$
(I+R) f(r z)=C \int_{\mathbf{B}^{n}} \frac{\left(1-|y|^{2}\right)^{N}(I+R) f(y)}{(1-r z \bar{y})^{n+1+N}} d v(y)
$$

Next, there is a constant $C>0$ such that for any $0<r<1, z, y \in \mathbf{B}^{n}$, $|1-r z \bar{y}| \geq C|1-z \bar{y}|$, and the above formula gives that

$$
|(I+R) f(r z)| \preceq \int_{\mathbf{B}^{n}} \frac{\left(1-|y|^{2}\right)^{N}|(I+R) f(y)|}{|1-z \bar{y}|^{n+1+N}} d v(y) .
$$

Combining the above results we have that

$$
\begin{aligned}
& \chi_{D_{\alpha}(\zeta)}(z)\left(1-|z|^{2}\right)|(I+R) f(r z)| \\
& \quad \preceq \chi_{D_{\alpha}(\zeta)}(z) \int_{\mathbf{B}^{n}} \frac{\left(1-|y|^{2}\right)^{N-1}\left(1-|z|^{2}\right)\left(\left(1-|y|^{2}\right)|(I+R) f(y)|\right)}{|1-z \bar{y}|^{n+1+N}} d v(y) \\
& \quad=C \chi_{D_{\alpha}(\zeta)}(z) P^{N-1,1}\left(\left(1-|\cdot|^{2}\right)(I+R) f\right)(z):=G(z, \zeta) .
\end{aligned}
$$

Theorem 2.8 shows that provided $N$ is chosen sufficiently large, $P^{N-1,1}$ maps $F^{\alpha, p, 2}(w)$ to itself, and in particular that

$$
\begin{aligned}
& \|G\|_{L^{p}(w)\left(L^{2}\left(\frac{d v(z)}{\left(1-|z|^{2}\right)^{n+1}}\right)\right)}=\left\|P^{N-1,1}\left(\left(1-|\cdot|^{2}\right)(I+R) f\right)\right\|_{\alpha, p, 2, w} \\
& \preceq\left\|\left(1-|\cdot|^{2}\right)(I+R) f\right\|_{\alpha, p, 2, w}=C\left\|A_{\alpha, 1,2,0}(f)\right\|_{L^{p}(w)}<+\infty .
\end{aligned}
$$

Consequently

$$
\left\|f_{r}\right\|_{H^{p}(w)} \preceq\left\|A_{\alpha, 1,2,0}(f)\right\|_{L^{p}(w)},
$$

and therefore $f \in H^{p}(w)$.

We will now remark on some facts about weighted Hardy-Sobolev spaces. Let us recall, that if $1<p<+\infty, 0<s<n$, and $w$ is an $A_{p}$-weight, we denote by $H_{s}^{p}(w)$ the space of holomorphic functions $f$ on $\mathbf{B}^{n}$ satisfying that

$$
\|f\|_{H_{s}^{p}(w)}=\left\|(I+R)^{s} f\right\|_{H^{p}(w)}<+\infty .
$$

The results obtained in the previous theorems give alternative equivalent definitions of the spaces $H_{s}^{p}(w)$ in terms of admissible maximal or radial functions and admissible area functions. 
On the other hand, when $w \equiv 1$, and $0<s<n$, it is well known, see for instance [CaOr1], that the space $H_{s}^{p}$ admits a representation in terms of a fractional Cauchy-type kernel $C_{s}$ defined by

$$
C_{s}(z, \zeta)=\frac{1}{(1-z \bar{\zeta})^{n-s}} .
$$

The same lines of the proof of the unweighted case can be used to obtain a similar characterization in the weighted case. We just have to use that the Hardy-Littlewood maximal operator is bounded in $L^{p}(w)$, if $w$ is an $A_{p}$-weight and Lemma 2.1.

TheOREM 2.13. Let $1<p<+\infty, 0<s<n$, and $w$ be an $A_{p}$-weight. We then have that the map

$$
C_{s}(f)(z)=\int_{\mathbf{B}^{n}} \frac{f(\zeta)}{(1-z \bar{\zeta})^{n-s}} d \sigma(\zeta)
$$

is a bounded map of $L^{p}(w)$ onto $H_{s}^{p}(w)$.

\section{§3. Holomorphic potentials and Carleson measures}

In this section we will study Carleson measures for $H_{s}^{p}(w), 1<p<+\infty$ and $0<s<n$, that is, the positive finite Borel measures $\mu$ on $\mathbf{B}^{n}$ satisfying

$$
\|f\|_{L^{p}(d \mu)} \leq C\|f\|_{H_{s}^{p}(w)}, \quad f \in L^{p}(w) .
$$

In what follows we will write

$$
\chi_{E} w d \sigma=\frac{1}{|E|} \int_{E} w
$$

where $E$ is a measurable set in $\mathbf{S}^{n}$ and $|E|$ denotes its Lebesgue measure.

By Theorem 2.13, this inequality can be rewritten as follows:

$$
\left\|C_{s}(f)\right\|_{L^{p}(d \mu)} \leq C\|f\|_{L^{p}(w)}, \quad f \in L^{p}(w) .
$$

We recall that we have defined the non-isotropic potential of a positive Borel function $f$ on $\mathbf{S}^{n}$ by

$$
K_{s}(f)(z)=\int_{\mathbf{S}^{n}} K_{s}(z, \zeta) f(\zeta) d \sigma(\zeta)=\int_{\mathbf{S}^{n}} \frac{f(\zeta)}{|1-z \bar{\zeta}|^{n-s}} d \sigma(\zeta)
$$

for $z \in \overline{\mathbf{B}}^{n}$. 
Analogously to what happens for isotropic potentials (see [Ad]), in the nonisotropic case it can be proved that if $w$ is an $A_{p}$ weight and $\zeta_{0} \in \mathbf{S}^{n}$ satisfies that

$$
\int_{\mathbf{S}^{n}} \frac{1}{\left|1-\zeta_{0} \bar{\zeta}\right|^{(n-s) p^{\prime}}} w^{-\left(p^{\prime}-1\right)}(\zeta) d \sigma(\zeta)<+\infty
$$

then for any $f \in L^{p}(w), K_{s}(f)$ is continuous in $\zeta_{0}$. Observe that when $w \equiv 1$, (3.4) holds if and only if $n-s p<0$. In the general weighted case, if $w$ satisfies a doubling condition of order $\tau$, and $\tau-s p<0$, we also have that (3.4) holds, and consequently the Carleson measures in this case for weighted Hardy Sobolev spaces are just the finite ones. Indeed, assume that $\tau-s p<0$. We then have

$$
\begin{aligned}
& \int_{\mathbf{S}^{n}} \frac{1}{\left|1-\zeta_{0} \bar{\zeta}\right|^{(n-s) p^{\prime}}} w^{-\left(p^{\prime}-1\right)}(\zeta) d \sigma(\zeta) \\
& =\int_{\mathbf{S}^{n}} w^{-\left(p^{\prime}-1\right)}(\zeta) \int_{\left|1-\zeta_{0} \bar{\zeta}\right|<t} \frac{d t}{t^{(n-s) p^{\prime}}} d \sigma(\zeta) \leq \int_{0}^{K} \frac{\int_{B\left(\zeta_{0}, t\right)} w^{-\left(p^{\prime}-1\right)}}{t^{(n-s) p^{\prime}}} \\
& \simeq \int_{0}^{K} \frac{t^{n} d t}{\left(X_{B\left(\zeta_{0}, t\right)} w\right)^{p^{\prime}-1} t^{(n-s) p^{\prime}}} \\
& \sum_{k} \frac{2^{-k s p^{\prime}}}{W\left(B\left(\zeta_{0}, 2^{-k}\right)\right)}
\end{aligned}
$$

The fact that $w$ satisfies condition $D_{\tau}$ gives that $W\left(\mathbf{S}^{n}\right) \preceq 2^{k \tau} W\left(B\left(\zeta_{0}, 2^{-k}\right)\right)$, and consequently the above sum is bounded, up to constants, by

$$
\sum_{k} 2^{k\left(\tau\left(p^{\prime}-1\right)-s p^{\prime}\right)}
$$

Since $\tau-s p<0$ we also have that $\tau\left(p^{\prime}-1\right)-s p^{\prime}<0$, and we are done.

From now on we will assume that $\tau-s p \geq 0$.

The problem of characterizing the positive finite Borel measures $\mu$ on $\mathbf{B}^{n}$ for which the following inequality holds

$$
\left\|K_{s}(f)\right\|_{L^{p}(d \mu)} \leq C\|f\|_{L^{p}(w)},
$$

has been thoroughly studied, and there are, among others, characterizations in terms of weighted nonisotropic Riesz capacities that are defined as follows: if $E \subset \mathbf{S}^{n}, 1<p<+\infty$ and $0<s<n$,

$$
C_{s p}^{w}(E)=\inf \left\{\|f\|_{L^{p}(w)}^{p} ; f \geq 0, K_{s}(f) \geq 1 \text { on } E\right\}
$$


It is well known, that when $w \equiv 1, C_{s p}(B(\zeta, r)) \simeq r^{n-s p}, \zeta \in \mathbf{S}^{n}, r<1$. See [Ad] for expressions of weighted capacities of balls in $\mathbf{R}^{n}$.

As it happens in $\mathbf{R}^{n}$ (see $[\mathrm{Ad}]$ ), we have that if $0 \leq n-s p,(3.5)$ holds if and only if there exists $C>0$ such that for any open set $G \subset \mathbf{S}^{n}$,

$$
\mu(T(G)) \leq C C_{s p}^{w}(G) .
$$

Here $T(G)$ is the admissible tent over $G$, defined by

$$
T(G)=T_{\alpha}(G)=\left(\bigcup_{\zeta \notin G} D_{\alpha}(\zeta)\right)^{c} .
$$

The problem of characterizing the Carleson measures $\mu$ for the holomorphic case (3.2) is much more complicated, even in the nonweighted case. Since $\left|C_{s}(z, \zeta)\right| \leq K_{s}(z, \zeta)$, it follows from Theorem 2.13, that (3.5) implies (3.2), and consequently that if condition (3.6) is satisfied, then $\mu$ is a Carleson measure for $H_{s}^{p}(w)$. Of course, when $n-s<1$ both problems are equivalent, even in the weighted case, simply because if $f \geq 0$, $\left|C_{s}(f)\right| \simeq K_{s}(f)$, but when $n>1$ (see [Ah] and [CaOr2]), condition (3.5) for the unweighted case is not, in general, equivalent to condition (3.2). Observe that when $n-s p \leq 0, H_{s}^{p}$ consists of regular functions, and consequently any finite measure is a Carleson measure for the holomorphic and the real case. It is proved in [CohVe1] that this equivalence still remains true if we are not too far from the regular case, namely, if $0 \leq n-s p<1$. The main purpose of this section is to obtain a result in this line for a wide class of $A_{p}$-weights.

In $[\mathrm{Ah}]$ it is also shown that if (3.2) holds for $w \equiv 1$, then the capacity condition on balls is satisfied, i.e. there exists $C>0$ such that $\mu(T(B(\zeta, r))) \leq C r^{n-s p}$, for any $\zeta \in \mathbf{S}^{n}$ and any $0<r<1$. The following proposition obtains a necessary condition in this line for the weighted holomorphic trace inequality.

Proposition 3.1. Let $1<p<+\infty, 0<s<n$. Let $\mu$ be a positive finite Borel measure on $\mathbf{B}^{n}$, and $w$ be an $A_{p}$-weight. Assume that there exists $C>0$ such that

$$
\|f\|_{L^{p}(d \mu)} \leq C\|f\|_{H_{s}^{p}(w)},
$$

for any $f \in H_{s}^{p}(w)$. We then have that there exists $C>0$ such that for any $\zeta \in \mathbf{S}^{n}, r>0$,

$$
\mu\left(T(B(\zeta, r)) \leq C \frac{W(B(\zeta, r))}{r^{s p}} .\right.
$$


Proof of Proposition 3.1. Let $\zeta \in \mathbf{S}^{n}, 0<r<1$ be fixed. If $z \in \overline{\mathbf{B}}^{n}$, let

$$
F(z)=\frac{1}{(1-(1-r) z \bar{\zeta})^{N}},
$$

with $N>0$ to be chosen later. If $z \in T(B(\zeta, r))$, and $z_{0}=z /|z|,(1-|z|) \preceq r$ and $\left|1-z_{0} \bar{\zeta}\right| \preceq r$. Hence $|1-(1-r) z \bar{\zeta}| \preceq r$, and consequently,

$$
\frac{\mu(T(B(\zeta, r)))}{r^{N p}} \leq C \int_{T(B(\zeta, r))}|F(z)|^{p} d \mu(z) .
$$

On the other hand,

$$
\begin{aligned}
\|F\|_{H_{s}^{p}(w)}^{p} & \leq C \int_{\mathbf{S}^{n}} \frac{1}{|1-(1-r) \eta \bar{\zeta}|^{(N+s) p}} w(\eta) d \sigma(\eta) \\
= & \int_{B(\zeta, r)} \frac{1}{|1-(1-r) \eta \bar{\zeta}|^{(N+s) p}} w(\eta) d \sigma(\eta) \\
& +\sum_{k \geq 1} \int_{B\left(\zeta, 2^{k+1} r\right) \backslash B\left(\zeta, 2^{k} r\right)} \frac{1}{|1-(1-r) \eta \bar{\zeta}|^{(N+s) p}} w(\eta) d \sigma(\eta) .
\end{aligned}
$$

If $k \geq 1$, and $\eta \in B\left(\zeta, 2^{k+1} r\right) \backslash B\left(\zeta, 2^{k} r\right),|1-(1-r) \eta \bar{\zeta}| \simeq 2^{k} r$. This estimates together with the fact that $w$ is doubling, give that the above is bounded by

$$
\sum_{k \geq 0} \frac{W\left(B\left(\zeta, 2^{k+1} r\right)\right)}{\left(2^{k} r\right)^{(N+s) p}} \preceq \frac{W(B(\zeta, r))}{r^{(N+s) p}} \sum_{k \geq 0}\left(\frac{C}{2^{(N+s) p}}\right)^{k},
$$

which gives the desired estimate, provided $N$ is chosen big enough.

We observe that for some special weights besides the case $w \equiv 1$, the expression that appears in the above proposition $W(B(\zeta, r)) / r^{s p}$ coincide with the weighted capacity of a ball (see $[\mathrm{Ad}]$ ).

If $\nu$ is a positive Borel measure on $\mathbf{S}^{n}, 1<p<+\infty, 0<s<n$ and $w$ is an $A_{p}$-weight, it is introduced in $[\mathrm{Ad}]$ the $(s, p)$-energy of $\nu$ with weight $w$, which is defined by

$$
\mathcal{E}_{s p}^{w}(\nu)=\int_{\mathbf{S}^{n}}\left(K_{s}(\nu)(\zeta)\right)^{p^{\prime}} w(\zeta)^{-\left(p^{\prime}-1\right)} d \sigma(\zeta)
$$

If we write $\left(K_{s}(\nu)\right)^{p^{\prime}}=\left(K_{s}(\nu)\right)^{p^{\prime}-1} K_{s}(\nu)$, Fubini's theorem gives that

$$
\mathcal{E}_{s p}^{w}(\nu)=\int_{\mathbf{S}^{n}} \mathcal{U}_{s p}^{w}(\nu)(\zeta) d \nu(\zeta)
$$


where

$$
\mathcal{U}_{s p}^{w}(\zeta)=K_{s}\left(w^{-1} K_{s}(\nu)\right)^{p^{\prime}-1}(\zeta)
$$

is the weighted nonlinear potential of the measure $\nu$. When $w \equiv 1$, Wolff's theorem (see $[\mathrm{HeWo}]$ ) gives another representation of the energy, in terms of the so-called Wolff's potential.

In the general case, it is introduced in [Ad] a weighted Wolff-type potential of a measure $\nu$ as

$$
\begin{aligned}
& \mathcal{W}_{s p}^{w}(\nu)(\zeta) \\
& =\int_{0}^{1}\left(\frac{\nu(B(\zeta, 1-r))}{(1-r)^{n-s p}}\right)^{p^{\prime}-1} \int_{B(\zeta, 1-r)} w^{-\left(p^{\prime}-1\right)}(\eta) d \sigma(\eta) \frac{d r}{1-r} .
\end{aligned}
$$

In the same paper, it is shown that provided $w$ is an $A_{p}$-weight, the following weighted Wolff-type theorem holds:

$$
\mathcal{E}_{s p}^{w}(\nu) \simeq \int_{\mathbf{S}^{n}} \mathcal{W}_{s p}^{w}(\nu)(\zeta) d \nu(\zeta)
$$

In fact, we have the pointwise estimate $\mathcal{W}_{s p}^{w}(\nu)(\zeta) \leq C \mathcal{U}_{s p}^{w}(\nu)(\zeta)$, and Wolff's theorem gives that the converse is true, provided we integrate with respect to $\nu$.

In [Ad] a weighted extremal theorem for the weighted Riesz capacities it is also shown, namely, if $G \subset \mathbf{S}^{n}$ is open, there exists a positive capacitary measure $\nu_{G}$ such that

(i) $\operatorname{supp} \nu_{G} \subset G$.

(ii) $\nu_{G}(G)=C_{s p}^{w}(G)=\mathcal{E}_{s p}^{w}\left(\nu_{G}\right)$.

(iii) $\mathcal{W}_{s p}^{w}\left(\nu_{G}\right)(\zeta) \geq C$, for $C_{s p}^{w}$-a.e. $\zeta \in G$.

(iv) $\mathcal{W}_{s p}^{w}\left(\nu_{G}\right)(\zeta) \leq C$, for any $\zeta \in \operatorname{supp} \nu_{G}$.

We now introduce two holomorphic weighted Wolff-type potentials, which generalize the ones defined in [CohVe1]. These potentials will be used in the proof of the characterization of the Carleson measures for $H_{s}^{p}(w)$, for the case $0 \leq \tau-s p<1$. Let $1<p<+\infty, 0<s<n / p$, and $\nu$ be a positive Borel measure on $\mathbf{S}^{n}$. For any $\lambda>0$, and $z \in \mathbf{B}^{n}$, we set

$$
\begin{aligned}
\mathcal{U}_{s p}^{w \lambda}(\nu)(z)=\int_{0}^{1} & \int_{\mathbf{S}^{n}}\left(\frac{\nu(B(\zeta, 1-r))}{(1-r)^{n-s p}}\right)^{p^{\prime}-1} \frac{(1-r)^{\lambda-n}}{(1-r z \bar{\zeta})^{\lambda}} \\
& \times\left(\int_{B(\zeta, 1-r)} w^{-\left(p^{\prime}-1\right)}\right) d \sigma(\zeta) \frac{d r}{1-r},
\end{aligned}
$$


and

$$
\begin{aligned}
\mathcal{V}_{s p}^{w \lambda}(\nu)(z)=\int_{0}^{1} & \left(\int_{\mathbf{S}^{n}} \frac{(1-r)^{\lambda+s p-n}}{(1-r z \bar{\zeta})^{\lambda}}\right. \\
& \left.\times\left(\int_{B(\zeta, 1-r)} w^{-\left(p^{\prime}-1\right)}\right)^{\frac{1}{p^{\prime}-1}} d \nu(\zeta)\right)^{p^{\prime}-1} \frac{d r}{1-r}
\end{aligned}
$$

Obviously, both potentials are holomorphic functions in the unit ball. We will see, that if $p \leq 2$ the first one is bounded from below by the weighted Wolff-type potential we have just introduced, whereas if $p \geq 2$, the second one is bounded from below by the same potential.

In the unweighted case, [CohVe1] the proof of the estimates of the holomorphic potentials, rely on an extension of Wolff's theorem. This extension gives that if $1<p<+\infty, s>0,0<q<+\infty$, and $\nu$ is a positive Borel measure on $\mathbf{S}^{n}$, then

$$
\int_{\mathbf{S}^{n}}\left(\int_{0}^{1}\left(\frac{\nu(B(\zeta, t))}{t^{n-s}}\right)^{q} \frac{d t}{t}\right)^{p^{\prime} / q} d \sigma(\zeta) \preceq \int_{\mathbf{S}^{n}} \mathcal{W}_{s p}^{w}(\nu)(\zeta) d \nu(\zeta) .
$$

Observe that if the above estimate holds for one $q_{0}$, it also holds for any $q \geq q_{0}$. The case $q=1$ is the integral estimate in Wolff's theorem, since we have that

$$
\mathcal{E}_{s p}(\nu) \simeq \int_{\mathbf{S}^{n}}\left(\int_{0}^{1} \frac{\nu(B(\zeta, t))}{t^{n-s}} \frac{d t}{t}\right)^{p^{\prime}} d \sigma(\zeta)
$$

The arguments in [CohVe1] can easily be used to show the following weighted version of the above theorem. We omit the details of the proof.

TheOREM 3.2. Let $1<p<+\infty$, w an $A_{p}$ weight, $s>0, K>0$, $0<q<+\infty$, and $\nu$ be a positive Borel measure on $\mathbf{S}^{n}$. Then

$$
\begin{aligned}
& \int_{\mathbf{S}^{n}}\left(\int_{0}^{K}\left(\frac{\nu(B(\zeta, t))}{t^{n-s}}\left(\int_{B(\zeta, t)} w^{-\left(p^{\prime}-1\right)}(\eta) d \sigma(\eta)\right)^{\frac{1}{p^{\prime}-1}}\right)^{q} \frac{d t}{t}\right)^{p^{\prime} / q} w(\zeta) d \sigma(\zeta) \\
& \preceq \int_{\mathbf{S}^{n}} \mathcal{W}_{s p}^{w}(\nu)(\zeta) d \nu(\zeta) .
\end{aligned}
$$

Before we obtain estimates of the $H_{s}^{p}(w)$-norm of the weighted holomorphic potentials already introduced, we will give a characterization for weights satisfying a doubling condition 
Lemma 3.3. Let $1<p<+\infty$ and $w$ be an $A_{p}$ weight on $\mathbf{S}^{n}$, and assume that $w \in D_{\tau}$, for some $\tau>0$. We then have:

(i) For any $t \in \mathbf{R}$ satisfying that $t>\tau-n$, there exists $C>0$ such that

$$
\int_{r}^{+\infty} \frac{1}{x^{t}} \int_{B(\zeta, x)} w \frac{d x}{x} \leq C \frac{1}{r^{t}} \oint_{B(\zeta, r)} w
$$

$r<1, \zeta \in \mathbf{S}^{n}$

(ii) For any $t \in \mathbf{R}$ satisfying that $t>\tau-n$, there exists $C>0$ such that

$$
\int_{0}^{r} x^{t}\left(\oint_{B(\zeta, x)} w^{-\left(p^{\prime}-1\right)}\right)^{p-1} \frac{d x}{x} \leq C r^{t}\left(\oint_{B(\zeta, r)} w^{-\left(p^{\prime}-1\right)}\right)^{p-1}
$$

$r<1, \zeta \in \mathbf{S}^{n}$

Proof of Lemma 3.3. We begin with the proof of part (i). Let $t>\tau-n$. Then

$$
\begin{aligned}
& \int_{r}^{+\infty} \frac{1}{x^{t}} \int_{B(\zeta, x)} w \frac{d x}{x}=\sum_{k \geq 0} \int_{2^{k} r}^{2^{k+1} r} \frac{1}{x^{t}} \int_{B(\zeta, x)} w \frac{d x}{x} \\
& \preceq \sum_{k \geq 0} \frac{1}{2^{k(t+n)} r^{t+n}} W\left(B\left(\zeta, 2^{k+1} r\right)\right) \preceq \sum_{k \geq 0} \frac{1}{2^{k(t+n)} r^{t+n}} 2^{k \tau} W(B(\zeta, r)) \\
& =C \frac{1}{r^{\delta}} \int_{B(\zeta, r)} w
\end{aligned}
$$

since $w$ is in $D_{\tau}$, and $t+n>\tau$.

Next we show that (ii) holds. If $\zeta \in \mathbf{S}^{n}$ and $r>0$, the fact that $w \in A_{p}$ gives that $\left(X_{B(\zeta, x)} w^{-\left(p^{\prime}-1\right)}\right)^{p-1} \simeq\left(X_{B(\zeta, x)} w\right)^{-1}$, and consequently,

$$
\begin{aligned}
& \int_{0}^{r} x^{t}\left(\int_{B(\zeta, x)} w^{-\left(p^{\prime}-1\right)}\right)^{p-1} \frac{d x}{x}=\sum_{k \geq 0} \int_{2^{-k} r}^{2^{-k+1} r} x^{t}\left(\int_{B(\zeta, x)} w^{-\left(p^{\prime}-1\right)}\right)^{p-1} \frac{d x}{x} \\
& \preceq \sum_{k \geq 0} 2^{-k t} r^{t} \frac{1}{X_{B\left(\zeta, 2^{-k} r\right)} w} \preceq \sum_{k \geq 0} \frac{1}{2^{k(t+n)}} r^{t-n} 2^{k \tau} W(B(\zeta, r)) \\
& \simeq r^{t}\left(\int_{B(\zeta, r)} w^{-\left(p^{\prime}-1\right)}\right)^{p-1} \cdot
\end{aligned}
$$


Remark. In fact, it can be proved that both conditions (i) and (ii) are in turn equivalent to the fact that the $A_{p}$ weight is in $D_{\tau}$.

We can now obtain the estimates on the weighted holomorphic potentials defined in (3.10) and (3.11).

TheOrem 3.4. Let $1<p<+\infty, 0<\alpha<n, w$ an $A_{p}$-weight. Assume that $w$ is in $D_{\tau}$ for some $0 \leq \tau-s p<1$. We then have:

(1) If $1<p<2$, there exists $0<\lambda<1$ and $C>0$ such that for any finite positive Borel measure $\nu$ on $\mathbf{S}^{n}$ the following assertions hold:

a) For any $\eta \in \mathbf{S}^{n}$,

$$
\lim _{\rho \rightarrow 1} \operatorname{Re} \mathcal{U}_{s p}^{w \lambda}(\nu)(\rho \eta) \geq C \mathcal{W}_{s p}^{w \lambda}(\nu)(\eta)
$$

b) $\left\|\mathcal{U}_{s p}^{w \lambda}(\nu)\right\|_{H_{s}^{p}(w)}^{p} \leq C \mathcal{E}_{s p}^{w}(\nu)$.

(2) If $p \geq 2$, there exists $0<\lambda<1$ and $C>0$ such that for any finite positive Borel measure $\nu$ on $\mathbf{S}^{n}$ the following assertions hold:

a) For any $\eta \in \mathbf{S}^{n}$,

$$
\lim _{\rho \rightarrow 1} \operatorname{Re} \mathcal{V}_{s p}^{w \lambda}(\nu)(\rho \eta) \geq C \mathcal{W}_{s p}^{w \lambda}(\nu)(\eta)
$$

b) $\left\|\mathcal{V}_{s p}^{w \lambda}(\nu)\right\|_{H_{s}^{p}(w)}^{p} \leq C \mathcal{E}_{s p}^{w}(\nu)$

Proof of Theorem 3.4. We will follow the scheme of [CohVe1] where it is proved for the unweighted case. The weights introduce new technical difficulties that require a careful use of the hypothesis $A_{p}$ and $D_{\tau}$ that we assume on the weight $w$. In order to make the proof easier to follow we sketch some of the arguments in [CohVe1], emphasizing the necessary changes we need to make in the weighted case.

Let us prove (1). We choose $\lambda$ such that $\tau-s p<\lambda<1$ and define $\mathcal{U}_{s p}^{w \lambda}$ as in 3.10. Then $\tau-s<\frac{\lambda+s-\tau(2-p)}{p-1}$. Consequently there exists $t$ such that $\tau-s<t<\frac{\lambda+s-\tau(2-p)}{p-1}$. Observe that $t+s-n>\tau-n$ and $\frac{\lambda+s-t(p-1)}{2-p}-n>\tau-n$. 
We begin now the proof of a). The fact that $\lambda<1$ gives that if $\rho<1$, $\eta \in \mathbf{S}^{n}$, and $C>0$,

$$
\begin{aligned}
\operatorname{Re}_{s p}^{w \lambda}(\rho \eta) \succeq \int_{0}^{1} & \int_{B(\eta, C(1-r))}\left(\frac{\nu(B(\zeta, 1-r))}{(1-r)^{n-s p}}\right)^{p^{\prime}-1} \frac{(1-r)^{\lambda-n}}{|1-r \rho \eta \bar{\zeta}|^{\lambda}} \\
& \times\left(\int_{B(\zeta, 1-r)} w^{-\left(p^{\prime}-1\right)}\right) d \sigma(\zeta) \frac{d r}{1-r}
\end{aligned}
$$

If $C>0$ has been chosen small enough, we have that for any $\zeta \in B(\eta, C(1-$ $r)), B(\eta, C(1-r)) \subset B(\zeta, 1-r)$. In addition, $|1-r \rho \eta \bar{\zeta}| \preceq|1-r \rho|$. These estimates, together with the fact that $w^{-\left(p^{\prime}-1\right)}$ satisfies a doubling condition, give that the above integral is bounded from below by

$$
\begin{aligned}
& C \int_{0}^{1} \int_{B(\eta, C(1-r))}\left(\frac{\nu(B(\eta, C(1-r)))}{(1-r)^{n-s p}}\right)^{p^{\prime}-1} \frac{(1-r)^{\lambda-n}}{\mid 1-r \rho^{\lambda}} \\
& \quad \times\left(\int_{B(\eta, 1-r)} w^{-\left(p^{\prime}-1\right)}\right) d \sigma(\zeta) \frac{d r}{1-r} \\
& \geq C \int_{0}^{\rho}\left(\frac{\nu(B(\eta, C(1-r)))}{(1-r)^{n-s p}}\right)^{p^{\prime}-1} \frac{(1-r)^{\lambda}}{|1-r \rho|^{\lambda}}\left(\int_{B(\eta, 1-r)} w^{-\left(p^{\prime}-1\right)}\right) \frac{d r}{1-r} \\
& \geq C \int_{0}^{\rho}\left(\frac{\nu(B(\eta, C(1-r)))}{(1-r)^{n-s p}}\right)^{p^{\prime}-1}\left(\int_{B(\eta, 1-r)} w^{-\left(p^{\prime}-1\right)}\right) \frac{d r}{1-r},
\end{aligned}
$$

where in last estimate we have used that since $r<\rho, 1-r \rho \simeq 1-r$.

We have proved then

$$
\int_{0}^{\rho}\left(\frac{\nu(B(\eta, C(1-r)))}{(1-r)^{n-s p}}\right)^{p^{\prime}-1}\left(\int_{B(\eta, 1-r)} w^{-\left(p^{\prime}-1\right)}\right) \frac{d r}{1-r} \leq C \operatorname{Re} \mathcal{U}_{s p}^{w \lambda}(\nu)(\rho \eta)
$$

and letting $\rho \rightarrow 1$, we obtain a).

In order to obtain the norm estimate, lets us simply write $\mathcal{U}(z)=$ $\mathcal{U}_{s p}^{w \lambda}(\nu)(z)$, and prove that for $k>s$,

$$
\begin{aligned}
& \|\mathcal{U}\|_{H F_{s}^{p 1}(w)}^{p} \\
& \quad=|\mathcal{U}(0)|^{p}+\int_{\mathbf{S}^{n}}\left(\int_{0}^{1}(1-\rho)^{k-s}\left|(I+R)^{k} \mathcal{U}(\rho \eta)\right| \frac{d \rho}{1-\rho}\right)^{p} w(\eta) d \sigma(\eta) \\
& \quad \leq C \mathcal{E}_{s p}^{w}(\nu) .
\end{aligned}
$$


But

$$
\begin{aligned}
& \int_{0}^{1}(1-\rho)^{k-s}\left|(I+R)^{k} \mathcal{U}(\rho \eta)\right| \frac{d \rho}{1-\rho} \\
& \preceq \int_{0}^{1}(1-\rho)^{k-s} \int_{0}^{1} \int_{\mathbf{S}^{n}}\left(\frac{\nu(B(\zeta, 1-r))}{(1-r)^{n-s p}}\right)^{p^{\prime}-1} \frac{(1-r)^{\lambda-n}}{|1-\rho r \eta \bar{\zeta}|^{\lambda+k}} \\
& \quad \times\left(\int_{B(\zeta, 1-r)} w^{-\left(p^{\prime}-1\right)}\right) d \sigma(\zeta) \frac{d r}{1-r} \frac{d \rho}{1-\rho} \preceq \Upsilon(\eta),
\end{aligned}
$$

where

$$
\begin{aligned}
\Upsilon(\eta)=\int_{0}^{1} & \int_{\mathbf{S}^{n}}\left(\frac{\nu(B(\zeta, 1-r))}{(1-r)^{n-s p}}\right)^{p^{\prime}-1} \frac{(1-r)^{\lambda-n}}{|1-r \eta \bar{\zeta}|^{\lambda+s}} \\
& \times\left(\int_{B(\zeta, 1-r)} w^{-\left(p^{\prime}-1\right)}\right) d \sigma(\zeta) \frac{d r}{1-r}
\end{aligned}
$$

Observe that $|\mathcal{U}(0)|^{p} \leq C\|\Upsilon\|_{L^{p}(w)}^{p}$. Consequently, in order to finish the proof of the theorem, we just need to show that

$$
\|\Upsilon\|_{L^{p}(w)}^{p} \leq C \mathcal{E}_{s p}^{w}(\nu)
$$

Hölder's inequality with exponent $\frac{1}{p-1}>1$ gives that

$$
\Upsilon(\eta) \leq \Upsilon_{1}(\eta)^{p-1} \Upsilon_{2}(\eta)^{2-p}
$$

where

$$
\begin{aligned}
\Upsilon_{1}(\eta)=\int_{0}^{1} & \int_{\mathbf{S}^{n}} \frac{\nu(B(\zeta, 1-r))}{(1-r)^{n-s}} \frac{(1-r)^{t-n}}{|1-r \eta \bar{\zeta}|^{t}} \\
& \times\left(\int_{B(\zeta, 1-r)} w^{-\left(p^{\prime}-1\right)}\right)^{p-1} d \sigma(\zeta) \frac{d r}{1-r}
\end{aligned}
$$

and

$$
\begin{aligned}
\Upsilon_{2}(\eta)= & \int_{0}^{1} \int_{\mathbf{S}^{n}}\left(\frac{\nu(B(\zeta, 1-r))}{(1-r)^{n-s}}\right)^{p^{\prime}} \frac{(1-r)^{\frac{\lambda+s-t(p-1)}{2-p}-n}}{|1-r \eta \bar{\zeta}|^{\frac{\lambda+s-t(p-1)}{2-p}}} \\
& \times\left(\int_{B(\zeta, 1-r)} w^{-\left(p^{\prime}-1\right)}\right)^{p} \frac{d \sigma(\zeta) d r}{1-r}
\end{aligned}
$$


We begin estimating the function $\Upsilon_{1}$. If $\zeta \in B(\tau, 1-r)$, we have that $B(\zeta, 1-r) \subset B(\tau, C(1-r))$, and since $w^{-\left(p^{\prime}-1\right)}$ satisfies a doubling condition,

$$
\begin{gathered}
\Upsilon_{1}(\eta) \preceq \int_{0}^{1}(1-r)^{t-2 n+s} \int_{\mathbf{S}^{n}} \int_{B(\tau, C(1-r))} \frac{d \sigma(\zeta)}{|1-r \eta \bar{\zeta}|^{t}} \\
\times\left(\oint_{B(\tau, 1-r)} w^{-\left(p^{\prime}-1\right)}\right)^{p-1} \frac{d \nu(\tau) d r}{1-r} .
\end{gathered}
$$

Next, we observe that if $\zeta \in B(\tau, C(1-r)),|1-r \eta \bar{\tau}| \preceq|1-r \eta \bar{\zeta}|$. Hence, the above is bounded by

$$
C \int_{0}^{1}(1-r)^{t-n+s} \int_{\mathbf{S}^{n}} \frac{\left(X_{B(\tau, 1-r)} w^{-\left(p^{\prime}-1\right)}\right)^{p-1}}{|1-r \eta \bar{\tau}|^{t}} d \nu(\tau) \frac{d r}{1-r} .
$$

Since

$$
\begin{aligned}
& \int_{\mathbf{S}^{n}} \frac{\left(X_{B(\tau, 1-r)} w^{-\left(p^{\prime}-1\right)}\right)^{p-1}}{|1-r \eta \bar{\tau}|^{t}} d \nu(\tau) \\
& \preceq \int_{\mathbf{S}^{n}}\left(\chi_{B(\tau, 1-r)} w^{-\left(p^{\prime}-1\right)}\right)^{p-1} \int_{|1-r \eta \bar{\tau}| \leq \delta} \frac{d \delta}{\delta^{t+1}} d \nu(\tau),
\end{aligned}
$$

the above estimate, together with Fubini's theorem and the fact that $t-$ $n+s>\tau-n$ give that $\Upsilon_{1}(\eta)$ is bounded by

$$
\begin{aligned}
& C \int_{0}^{1} \int_{B(\eta, \delta)} \delta^{t-n+s}\left(\int_{B(\tau, \delta)} w^{-\left(p^{\prime}-1\right)}\right)^{p-1} d \nu(\tau) \frac{d \delta}{\delta^{t+1}} \\
& \preceq \int_{0}^{1}\left(\oint_{B(\eta, \delta)} w^{-\left(p^{\prime}-1\right)}\right)^{p-1} \frac{\nu(B(\eta, \delta))}{\delta^{n-s}} \frac{d \delta}{\delta},
\end{aligned}
$$

where we have used the fact that if $\tau \in B(\eta, \delta)$, then $B(\tau, \delta) \subset B(\eta, C \delta)$, for some $C>0$ and that $w^{-\left(p^{\prime}-1\right)}$ satisfies a doubling condition.

Applying Hölder's inequality with exponent $\frac{1}{(p-1)^{2}}>1$, we deduce that

$$
\begin{aligned}
\|\Upsilon\|_{L^{p}(w)} \preceq & \left(\int _ { \mathbf { S } ^ { n } } \left(\int_{0}^{1}\left(\int_{B(\eta, 1-r)} w^{-\left(p^{\prime}-1\right)}\right)^{p-1}\right.\right. \\
& \left.\left.\times \frac{\nu(B(\eta, \delta))}{\delta^{n-s}} \frac{d \delta}{\delta}\right)^{p^{\prime}} w d \sigma\right)^{(p-1)^{2}}\left(\int_{\mathbf{S}^{n}} \Upsilon_{2} w\right)^{p(2-p)} .
\end{aligned}
$$


Theorem 3.2 with $q=1$ gives that the first factor on the right is bounded by $C \mathcal{E}_{s p}^{w}(\nu)^{(p-1)^{2}}$.

Next we deal with the integral involving $\Upsilon_{2}$. We recall that $l=$ $\frac{\lambda+s-t(p-1)}{2-p}-n>\tau-n$. Fubini's theorem gives that

$$
\begin{aligned}
\int_{\mathbf{S}^{n}} \Upsilon_{2} w=\int_{\mathbf{S}^{n}} & \int_{0}^{1}\left(\frac{\nu(B(\zeta, 1-r))}{(1-r)^{n-s}}\right)^{p^{\prime}}(1-r)^{l}\left(\int_{B(\zeta, 1-r)} w^{-\left(p^{\prime}-1\right)}\right)^{p} \\
& \times \int_{\mathbf{S}^{n}} \frac{w(\eta) d \sigma(\eta)}{|1-r \eta \bar{\zeta}|^{l+n}} \frac{d \sigma(\zeta) d r}{1-r}
\end{aligned}
$$

But, as before, since $l>\tau-n$,

$$
\int_{\mathbf{S}^{n}} \frac{w(\eta) d \sigma(\eta)}{|1-r \eta \bar{\zeta}|^{l+n}} \leq \frac{C}{(1-r)^{l}} \oint_{B(\zeta, 1-r)} w .
$$

The above, together with Fubini's theorem gives that

$$
\begin{aligned}
\int_{\mathbf{S}^{n}} \Upsilon_{2} w \preceq \int_{0}^{1} & \int_{\mathbf{S}^{n}} \oint_{B(\eta, 1-r)}\left(\frac{\nu(B(\zeta, 1-r))}{(1-r)^{n-s}}\right)^{p^{\prime}} \\
& \times\left(\int_{B(\zeta, 1-r)} w^{-\left(p^{\prime}-1\right)}\right)^{p} d \sigma(\zeta) w(\eta) \frac{d \sigma(\eta) d r}{1-r} .
\end{aligned}
$$

But if $\zeta \in B(\eta, 1-r), B(\zeta, 1-r) \subset B(\eta, C(1-r))$, for some $C>0$, and in consequence the above is bounded by

$$
C \int_{\mathbf{S}^{n}} \int_{0}^{1}\left(\frac{\nu(B(\eta, C(1-r)))}{(1-r)^{n-s}}\right)^{p^{\prime}}\left(\int_{B(\eta, 1-r)} w^{-\left(p^{\prime}-1\right)}\right)^{p} \frac{d r}{1-r} w(\eta) d \sigma(\eta) .
$$

The change of variables $C(1-r)=y-1$ gives that we can estimate the previous expression by

$$
\begin{aligned}
& C \int_{\mathbf{S}^{n}} \int_{0}^{1}\left(\frac{\nu(B(\eta,(1-y)))}{(1-y)^{n-s}}\right)^{p^{\prime}}\left(\int_{B(\eta, 1-y)} w^{-\left(p^{\prime}-1\right)}\right)^{p} \frac{d y}{1-y} w(\eta) d \sigma(\eta) \\
& \quad+\nu\left(\mathbf{S}^{n}\right)^{p^{\prime}}\left(\int_{\mathbf{S}^{n}} w^{-\frac{1}{p-1}}\right)^{p}=I+I I .
\end{aligned}
$$

Theorem 3.2 gives that $I I \preceq C \mathcal{E}_{s p}^{w}(\nu)$, and Theorem 3.2 with $q=p^{\prime}$ gives that $I \leq C \mathcal{E}_{s p}^{w}(\nu)$. Consequently, $\int_{\mathbf{S}^{n}} \Upsilon_{2} w \leq C \mathcal{E}_{s p}^{w}(\nu)$, and plugging this estimate in (3.18), we deduce that

$$
\|\Upsilon\|_{L^{p}(w)}^{p} \preceq C \mathcal{E}_{s p}^{w}(\nu)^{(p-1)^{2}} \mathcal{E}_{s p}^{w}(\nu)^{p(2-p)} \simeq \mathcal{E}_{s p}^{w}(\nu) .
$$


We now sketch the proof of part (2). We choose $\lambda>0$ such that $\tau-s p<$ $\lambda<1$, and define $\mathcal{V}_{s p}^{w \lambda}(\nu)(z)$ as in (3.11). Let us simplify the notation and just write $\mathcal{V}(z)=\mathcal{V}_{s p}^{w \lambda}(\nu)(z)$. Let $\varepsilon \in \mathbf{R}$ such that $\tau<\varepsilon+n<\lambda+s p$.

The proof of a) is analogous to the one in case $1<p<2$.

For the proof of $\mathrm{b}$ ), let us consider $k>s$. It will be enough to prove the following:

$$
\begin{aligned}
& \|\mathcal{V}\|_{H F_{s}^{p 1}(w)}^{p} \\
& \quad=|\mathcal{V}(0)|^{p}+\int_{\mathbf{S}^{n}}\left(\int_{0}^{1}(1-\rho)^{k-s}\left|(I+R)^{k} \mathcal{V}(\rho \zeta)\right| \frac{d \rho}{1-\rho}\right)^{p} w(\zeta) d \sigma(\zeta) \\
& \leq C \mathcal{E}_{s p}^{w}(\nu) .
\end{aligned}
$$

Let us begin with the estimate $|\mathcal{V}(0)|^{p} \preceq \mathcal{E}_{s p}^{w}(\nu)$. If $p>2$, Hölder's inequality with exponent $\frac{1}{p^{\prime}-1}>1$, gives that

$$
\begin{aligned}
|\mathcal{V}(0)| \leq & \left(\int_{0}^{1} \int_{\mathbf{S}^{n}}(1-r)^{\varepsilon}\left(\int_{B(\zeta, 1-r)} w^{-\left(p^{\prime}-1\right)}\right)^{\frac{1}{p^{\prime}-1}} d \nu(\zeta) \frac{d r}{1-r}\right)^{p^{\prime}-1} \\
& \times\left(\int_{0}^{1}\left((1-r)^{\left(p^{\prime}-1\right)(\lambda+s p-n-\varepsilon)}\right)^{\frac{1}{2-p^{\prime}}} \frac{d r}{1-r}\right)^{2-p^{\prime}} \\
\preceq & \nu\left(\mathbf{S}^{n}\right)^{p^{\prime}-1} \int_{\mathbf{S}^{n}} w^{-\left(p^{\prime}-1\right)} .
\end{aligned}
$$

The case $p=2$ is proved similarly. Consequently, for any $p \geq 2$,

$$
|\mathcal{V}(0)|^{p} \preceq \nu\left(\mathbf{S}^{n}\right)^{p^{\prime}}\left(\oint_{\mathbf{S}^{n}} w^{-\left(p^{\prime}-1\right)}\right)^{p} \leq C \mathcal{E}_{s p}^{w}(\nu),
$$

where the constant $C$ may depend on $w$.

Following with the estimate of $\|\mathcal{V}\|_{H F_{s}^{p 1}(w)}$, we recall (for example see [CohVe2], Proposition 1.4) that if $k>0,0<\lambda<1$, and $z \in \mathbf{B}^{n}$,

$$
\left|(I+R)^{k}\left(\int_{\mathbf{S}^{n}} \frac{d \nu(\zeta)}{(1-z \bar{\zeta})^{\lambda}}\right)^{p^{\prime}-1}\right| \leq C\left(\int_{\mathbf{S}^{n}} \frac{d \nu(\zeta)}{|1-z \bar{\zeta}|^{\lambda}}\right)^{p^{\prime}-2} \int_{\mathbf{S}^{n}} \frac{d \nu(\zeta)}{|1-z \bar{\zeta}|^{\lambda+k}} .
$$

Plugging this estimate in (3.19) and using that $p^{\prime}-2 \leq 0$, we get

$$
\begin{aligned}
& \left|(I+R)^{k} \mathcal{V}(\rho \eta)\right| \preceq \int_{0}^{1} \int_{1-r<\delta, 1-\rho<\delta<3} \\
& \frac{(1-r)^{\left(p^{\prime}-1\right)(\lambda+s p-n)}\left(\int_{B(\eta, \delta)}\left(X_{B(\zeta, 1-r)} w^{\left.-\left(p^{\prime}-1\right)\right)^{\frac{1}{p^{\prime}-1}}} d \nu(\zeta)\right)^{p^{\prime}-1}\right.}{\delta^{\lambda+k+1+\left(p^{\prime}-2\right) \lambda}} \frac{d \delta d r}{1-r} .
\end{aligned}
$$


Assume first that $p>2$. Fubini's theorem and Hölder's inequality with exponent $\frac{1}{p^{\prime}-1}>1$, gives that the above is bounded by

$$
\begin{aligned}
& \int_{1-\rho}^{3}\left(\int_{1-r<\delta<3}(1-r)^{\varepsilon} \int_{B(\eta, \delta)}\left(\int_{B(\zeta, 1-r)} w^{-\left(p^{\prime}-1\right)}\right)^{\frac{1}{p^{\prime}-1}} d \nu(\zeta) \frac{d r}{1-r}\right)^{p^{\prime}-1} \\
& \quad \times\left(\int_{1-r<\delta<3}\left(\frac{(1-r)^{(\lambda+s p-n)\left(p^{\prime}-1\right)-\varepsilon\left(p^{\prime}-1\right)}}{\delta^{\lambda+k+1+\left(p^{\prime}-2\right) \lambda}}\right)^{\frac{1}{2-p^{\prime}}} \frac{d r}{1-r}\right)^{2-p^{\prime}} d \delta .
\end{aligned}
$$

Next, Fubini's theorem and the fact that $\varepsilon>\tau-n$ give that

$$
\begin{aligned}
& \int_{1-r<\delta}(1-r)^{\varepsilon} \int_{B(\eta, \delta)}\left(\int_{B(\zeta, 1-r)} w^{-\left(p^{\prime}-1\right)}\right)^{\frac{1}{p^{\prime}-1}} d \nu(\zeta) \frac{d r}{1-r} \\
& \preceq \int_{B(\eta, \delta)} \delta^{\varepsilon}\left(\int_{B(\zeta, \delta)} w^{-\left(p^{\prime}-1\right)}\right)^{\frac{1}{p^{\prime}-1}} d \nu(\zeta) .
\end{aligned}
$$

We also have that since $\lambda+s p-n-\varepsilon>0,(3.20)$ is bounded by

$$
\int_{1-\rho}^{3}\left(\int_{B(\eta, \delta)}\left(\int_{B(\zeta, \delta)} w^{-\left(p^{\prime}-1\right)}\right)^{\frac{1}{p^{\prime}-1}} d \nu(\zeta)\right)^{p^{\prime}-1} \frac{d \delta}{\delta^{(n-s p)\left(p^{\prime}-1\right)+k+1}} .
$$

For the case $p=2$, we obtain the same estimate, applying directly condition (3.14) on (3.20).

Integrating with respect to $\rho$, and applying Fubini's theorem we get

$$
\begin{aligned}
& \int_{0}^{1}(1-\rho)^{k-s}\left|(I+R)^{k} \mathcal{V}(\rho \eta)\right| \frac{d \rho}{1-\rho} \\
& \preceq \int_{0}^{3}\left(\int_{B(\eta, \delta)}\left(\int_{B(\zeta, \delta)} w^{-\left(p^{\prime}-1\right)}\right)^{\frac{1}{p^{\prime}-1}} d \nu(\zeta)\right)^{p^{\prime}-1} \frac{d \delta}{\delta^{(n-s)\left(p^{\prime}-1\right)+1}},
\end{aligned}
$$

since $(n-s p)\left(p^{\prime}-1\right)+s=(n-s)\left(p^{\prime}-1\right)$. If $\tau \in B(\zeta, \delta)$, and $\zeta \in B(\eta, \delta)$, we have that $\tau \in B(\eta, C \delta)$. The fact that $w^{-\left(p^{\prime}-1\right)}$ satisfies a doubling condition, gives that the last integral is bounded by

$$
C \int_{0}^{3}\left(\frac{\nu(B(\eta, \delta))}{\delta^{n-s}}\right)^{p^{\prime}-1} \int_{B(\eta, \delta)} w^{-\left(p^{\prime}-1\right)} \frac{d \delta}{\delta} .
$$

Applying Theorem 3.2 with exponent $q=p^{\prime}-1$, we finally obtain that $\int_{\mathbf{S}^{n}}\left(\int_{0}^{1}(1-\rho)^{k-s}\left|(I+R)^{k} \mathcal{V}(\rho \eta)\right| \frac{d \rho}{\rho}\right)^{p} w(\eta) d \sigma(\eta) \preceq \int_{\mathbf{S}^{n}} \mathcal{W}_{s p}^{w}(\nu)(\zeta) d \nu(\zeta)$. 
We can now state the characterization of the weighted Carleson measures.

TheOREm 3.5. Let $1<p<+\infty, 0<n-s p<1$, w an $A_{p}$-weight, and $\mu$ a finite positive Borel measure on $\mathbf{B}^{n}$. Assume that $w$ is in $D_{\tau}$ for some $0 \leq \tau-s p<1$. We then have that the following statements are equivalent:

(i) $\left\|K_{\alpha}(f)\right\|_{L^{p}(d \mu)} \leq C\|f\|_{L^{p}(w)}$.

(ii) $\|f\|_{L^{p}(d \mu)} \leq C\|f\|_{H_{s}^{p}(w)}$.

Proof of Theorem 3.5. Let us show first that (i) $\Rightarrow$ (ii). Theorem 2.13 gives that condition (ii) can be rewritten as

$$
\left\|C_{s}(g)\right\|_{L^{p}(d \mu)} \leq C\|g\|_{L^{p}(w)} .
$$

This fact together with the estimate $\left|C_{s}(f)\right| \leq C K_{s}(|f|)$ finishes the proof of the implication.

Assume now that (ii) holds. Since a measure $\mu$ on $\mathbf{B}^{n}$ satisfies (i) if and only if (see (3.6)) there exists $C>0$ such that for any open set $G \subset \mathbf{S}^{n}, \mu(T(G)) \leq C C_{s p}^{w}(G)$, we will check that this estimate holds. Let $G \subset \mathbf{S}^{n}$ be an open set, and let $\nu$ be the extremal measure for $C_{s p}^{w}(G)$. We then have that $\mathcal{W}_{s p}^{w}(\nu) \geq 1$ except on a set of $C_{s p}^{w}$-capacity zero, and $\int_{\mathbf{S}^{n}} \mathcal{W}_{s p}^{w}(\nu) d \nu \leq C C_{s p}^{w}(G)$. Let us check that the first estimate also holds for a.e. $x \in G$ (with respect to Lebesgue measure on $\mathbf{S}^{n}$ ). Indeed, if $A \subset \mathbf{S}^{n}$ satisfies that $C_{s p}^{w}(A)=0$, and $\varepsilon>0$, let $f \geq 0$ be a function such that $K_{s}(f) \geq 1$ on $A$ and $\int_{\mathbf{S}^{n}} f^{p} w \leq \varepsilon$. Since $L^{p}(w) \subset L^{p_{1}}(d \sigma)$, for some $1<p_{1}<p$, (see Lemma 2.1) we then have $\|f\|_{L^{p_{1}(d \sigma)}} \leq C\|f\|_{L^{p}(w)} \leq C \varepsilon^{1 / p}$. Thus $C_{s p_{1}}(A)=0$, and in particular $|A|=0$.

Following with the proof of the implication consider the holomorphic function on $\mathbf{B}^{n}$ defined by $F(z)=\mathcal{U}_{s p}^{w \lambda}(\nu)(z)$ if $1<p<2, F(z)=$ $\mathcal{V}_{s p}^{w \lambda}(\nu)(z)$, if $p \geq 2$ where $\lambda$ is as in Theorem 3.4. Theorem 3.4 and the fact that $\nu$ is extremal give that

$$
\lim _{r \rightarrow 1} \operatorname{Re} F(r \zeta) \geq C \mathcal{W}_{s p}^{w}(\nu)(\zeta) \geq C
$$

for a.e. $x \in G$ with respect to $C_{s p}^{w}$, and in consequence, for a.e. $x \in G$ with respect to Lebesgue measure on $G$. Hence, if $P$ is the Poisson-Szegö kernel

$$
|F(z)|=\left|P\left[\lim _{r \rightarrow 1} F(r \cdot)\right](z)\right| \geq\left|P\left[\operatorname{Re} \lim _{r \rightarrow 1} F(r \cdot)\right](z)\right| \geq C,
$$


for any $z \in T(G)$, and since we are assuming that (ii) holds, we obtain

$$
\mu(T(E)) \leq \int_{T(E)}|F(z)|^{p} d \mu(z) \leq C\|F\|_{H_{s}^{p}(w)}^{p} \leq C \mathcal{E}_{s p}^{w}(\nu) \leq C C_{s p}^{w}(G)
$$

We finish with an example which shows that, simmilarly to what happens if $w \equiv 1$, if $w \in D_{\tau}$ and $\tau-s p>1$, then the equivalence between (i) and (ii) in the previous theorem need not to be true.

Proposition 3.6. Let $n \geq 3, p=2$, and $\tau \geq 0,0<s$ such that $1+2 s<\tau<2 s+n-1$. Assume also that $n<\tau<n+1$. Then there exists $w \in A_{2} \cap D_{\tau}$ and a positive Borel measure $\mu$ on $\mathbf{S}^{n}$ such that $\mu$ is a Carleson measure for $H_{s}^{2}(w)$, but it is not Carleson for $K_{s}\left[L^{2}(w)\right]$.

Proof of Proposition 3.6. If $\varepsilon=\tau-n$, and $\zeta=\left(\zeta^{\prime}, \zeta_{n}\right) \in \mathbf{S}^{n}$, we consider the weight on $\mathbf{S}^{n}$ defined by $w(\zeta)=\left(1-\left|\zeta^{\prime}\right|^{2}\right)^{\varepsilon}$. A calculation gives that $w(z)=\left(1-|z|^{2}\right)^{\varepsilon} \in A_{2}$ if and only if $-1<\varepsilon<1$, which is our case. We also have that if $\zeta \in \mathbf{S}^{n}, R>0$ and $j \geq 0$, then $W\left(B\left(\zeta, 2^{j} R\right)\right) \simeq 2^{j \tau} W(B(\zeta, R))$, i.e. $w \in D_{\tau}$.

Next, any function in $H_{s}^{2}(w)$ can be written as $\int_{\mathbf{S}^{n}} \frac{f(\zeta)}{(1-z \bar{\zeta})^{n-s}} d \sigma(\zeta), f \in$ $L^{2}(w)$. It is then immediate to check that the restriction to $B^{n-1}$ of any such function can be written as

$$
\int_{\mathbf{B}^{n-1}} \frac{g\left(\zeta^{\prime}\right)\left(1-\left|\zeta^{\prime}\right|^{2}\right)^{-\varepsilon / 2}}{\left(1-z^{\prime} \overline{\zeta^{\prime}}\right)^{n-s}} d v\left(\zeta^{\prime}\right)
$$

with $g \in L^{2}(d v)$. This last space coincides (see for instance [Pe]) with the Besov space $B_{s-\frac{1}{2}-\frac{\varepsilon}{2}}^{2}\left(\mathbf{B}^{n-1}\right)=H_{s-\frac{1}{2}-\frac{\varepsilon}{2}}^{2}\left(\mathbf{B}^{n-1}\right)$.

Next, $n-1-\left(s-\frac{1}{2}-\frac{\varepsilon}{2}\right) 2=\tau-2 s>1$, and Proposition 3.1 in [CaOr2] gives that there exists a positive Borel measure $\mu$ on $\mathbf{B}^{n}$ which is Carleson for $H_{s-\frac{1}{2}-\frac{\varepsilon}{2}}^{2}\left(\mathbf{S}^{n-1}\right)$, but it fails to be Carleson for the space $K_{s-\frac{1}{2}-\frac{\varepsilon}{2}}\left[L^{2}(d \sigma)\right]$. Thus the operator

$$
f \longrightarrow \int_{\mathbf{S}^{n-1}} \frac{f(\zeta)}{|1-z \bar{\zeta}|^{n-1-\left(s-\frac{1}{2}-\frac{\varepsilon}{2}\right)}} d \sigma(\zeta),
$$

is not bounded from $L^{2}(d \sigma)$ to $L^{2}(d \mu)$. Duality gives that the operator

$$
g \longrightarrow \int_{\mathbf{B}^{n-1}} \frac{g(z)}{|1-z \bar{\zeta}|^{n-1-\left(s-\frac{1}{2}-\frac{\varepsilon}{2}\right)}} d \mu(z)
$$


is also not bounded from $L^{2}(d \mu)$ to $L^{2}(d \sigma)$. But if $g \geq 0, g \in L^{2}(d \mu)$, Fubini's theorem gives

$$
\begin{aligned}
& \left\|\int_{\mathbf{B}^{n-1}} \frac{g(z)}{|1-z \bar{\zeta}|^{n-1-\left(s-\frac{1}{2}-\frac{\varepsilon}{2}\right)}}\right\|_{L^{2}(d \sigma)}^{2} \\
& =\int_{\mathbf{S}^{n-1}}\left(\int_{\mathbf{B}^{n-1}} \frac{g(z)}{|1-z \bar{\zeta}|^{n-1-\left(s-\frac{1}{2}-\frac{\varepsilon}{2}\right)}} d \mu(z)\right)^{2} d \sigma(\zeta) \\
& =\int_{\mathbf{S}^{n-1}} \int_{\mathbf{B}^{n-1}} \frac{g(z)}{|1-z \bar{\zeta}|^{n-1-\left(s-\frac{1}{2}-\frac{\varepsilon}{2}\right)}} d \mu(z) \\
& \quad \times \int_{\mathbf{B}^{n-1}} \frac{g(w)}{|1-w \bar{\zeta}|^{n-1-\left(s-\frac{1}{2}-\frac{\varepsilon}{2}\right)}} d \mu(w) d \sigma(\zeta) \\
& \simeq \int_{\mathbf{B}^{n-1}} \int_{\mathbf{B}^{n-1}} \frac{g(z) g(w)}{|1-z \bar{w}|^{n-1-2\left(s-\frac{1}{2}-\frac{\varepsilon}{2}\right)}} d \mu(z) d \mu(w),
\end{aligned}
$$

where the last estimate holds since $n-1-2\left(s-\frac{1}{2}-\frac{\varepsilon}{2}\right)=\tau-2 s>0$. Consequently, we have that for the measure $\mu$, it does not hold that for any $g \in L^{2}(d \mu)$

$$
\int_{\mathbf{B}^{n-1}} \int_{\mathbf{B}^{n-1}} \frac{g(z) g(w)}{|1-z \bar{w}|^{n-2\left(s-\frac{\varepsilon}{2}\right)}} d \mu(z) d \mu(w) \leq C\|g\|_{L^{2}(d \mu)} .
$$

We next check that the failure of being a Carleson measure for $K_{s}\left[L^{2}(w)\right]$ can be also rewritten in the same terms. An argument similar to the previous one, gives that $\mu$ is not Carleson for $K_{s}\left[L^{2}(w)\right]$ if and only if the operator

$$
f \longrightarrow \int_{\mathbf{B}^{n-1}} \frac{f(z)}{|1-y \bar{z}|^{n-s}} d v(z)
$$

is not bounded from $L^{2}(w d v)$ to $L^{2}(d \mu)$. Equivalently, writing $f(z)=$ $h(z)\left(1-|z|^{2}\right)^{\varepsilon / 2}$, this last assertion holds if and only if the operator

$$
f \longrightarrow \int_{\mathbf{B}^{n-1}} \frac{f(z)\left(1-|z|^{2}\right)^{-\varepsilon / 2}}{|1-y \bar{z}|^{n-s}} d v(z)
$$

is not bounded from $L^{2}(d v)$ to $L^{2}(d \mu)$. But an argument as before, using duality and Fubini's theorem, gives that the fact that of the unboundedness of the operator can be rewritten in terms of (3.21). 


\section{REFERENCES}

[Ad] D. R. Adams, Weighted nonlinear potential theory, Trans. Amer. Math. Soc., 297 (1986), 73-94.

[AdHe] D. R. Adams and L. I. Hedberg, Function Spaces and Potential Theory, Springer-Verlag Berlin-Heidelberg-New York, 1996.

[Ah] P. Ahern, Exceptional sets for holomorphic Sobolev functions, Michigan Math. J., 35 (1988), 29-41.

[AhCo] P. Ahern and W. S. Cohn, Exceptional sets for Hardy-Sobolev spaces, Indiana Math. J., 39 (1989), 417-451.

[AhBrCa] P. Ahern, J. Bruna and C. Cascante, $H^{p}$-theory for generalized M-harmonic functions in the unit ball, Indiana Math. J., 45 (1996), 103-135.

[BeLo] J. Berg and J. Löfström, Interpolation Spaces, an Introduction, SpringerVerlag Berlin, 1976.

[CaOr1] C. Cascante and J. M. Ortega, Tangential-exceptional sets for Hardy-Sobolev spaces, Illinois J. Math., 39 (1995), 68-85.

[CaOr2] C. Cascante and J. M. Ortega, Carleson measures on spaces of Hardy-Sobolev type, Canadian J. Math., 47 (1995), 1177-1200.

[CohVe1] W. S. Cohn and I. E. Verbitsky, Trace inequalities for Hardy-Sobolev functions in the unit ball of $\mathbf{C}^{n}$, Indiana Univ. Math. J., 43 (1994), 1079-1097.

[CohVe2] W. S. Cohn and I. E. Verbitsky, Non-linear potential theory on the ball, with applications to exceptional and boundary interpolation sets, Michigan Math. J., 42 (1995), 79-97.

[CoiMeSt] R. R. Coifman, Y. Meyer and E. M. Stein, Some new function spaces and their applications to harmonic analysis, Journal of Funct. Anal., 62 (1985), 304-335.

[HeWo] L. I. Hedberg and Th. H. Wolff, Thin sets in nonlinear potential theory, Ann. Inst. Fourier (Grenoble), 33 (1983), 161-187.

[KaKo] H. Kang and H. Koo, Two-weighted inequalities for the derivatives of holomorphic functions and Carleson measures on the ball, Nagoya Math. J., 158 (2000), 107-131.

[KeSa] R. Kerman and E. T. Sawyer, The trace inequality and eigenvalue estimates for Schrödinger operators, Ann. Inst. Fourier, 36 (1986), 207-228.

$[\mathrm{Lu}] \quad$ D. H. Luecking, Representation and duality in weighted spaces of analytic functions, Indiana Univ. Math., 34 (1985), 319-336.

[Ma] V. G. Maz'ya, Sobolev Spaces, Berlin: Springer, 1985.

[OF] J. M. Ortega and J. Fabrega, Holomorphic Triebel-Lizorkin Spaces, J. Funct. Analysis, 151 (1997), 177-212.

[Pe] M. M. Peloso, Möbius invariant spaces on the unit ball, Michigan Math. J., 39 (1992), 509-537.

[Ru $\quad$ W. Rudin, Function Theory in the Unit Ball of $\mathbf{C}^{n}$, New York: Springer, 1980.

[St2] E. M. Stein, Boundary behavior of holomorphic functions of several complex variables, Princeton University Press, 1972.

[StrTo] J.-O. Strömberg and A. Torchinsky, Weighted Hardy Spaces, Lecture Notes in Math. 1381, Springer-Verlag, 1989. 


\section{Carme Cascante}

Departament de Matemàtica Aplicada i Anàlisi Facultat de Matemàtiques

Universitat de Barcelona

Gran Via 585, 08071 Barcelona

Spain

cascante@ub.edu

Joaquin M. Ortega

Departament de Matemàtica Aplicada i Anàlisi Facultat de Matemàtiques

Universitat de Barcelona

Gran Via 585, 08071 Barcelona

Spain

ortega@ub.edu 